\title{
TITLE:
}

\section{Paradoxical activation of c-Src as a drug-resistant mechanism}

\section{AUTHOR(S):}

Higuchi, Makio; Ishiyama, Kenichi; Maruoka, Masahiro; Kanamori, Ryosuke; Takaori-Kondo, Akifumi; Watanabe, Naoki

\section{CITATION:}

Higuchi, Makio ...[et al]. Paradoxical activation of c-Src as a drugresistant mechanism. Cell Reports 2021, 34(12): 108876.

\section{ISSUE DATE:}

2021-03-23

URL:

http://hdl.handle.net/2433/262322

\section{RIGHT:}

(C) 2021 The Authors. This is an open access article under the CC BY-NCND license (http://creativecommons.org/licenses/by-nc-nd/4.0/). 


\section{Paradoxical activation of c-Src as a drug-resistant mechanism}

\section{Graphical abstract}

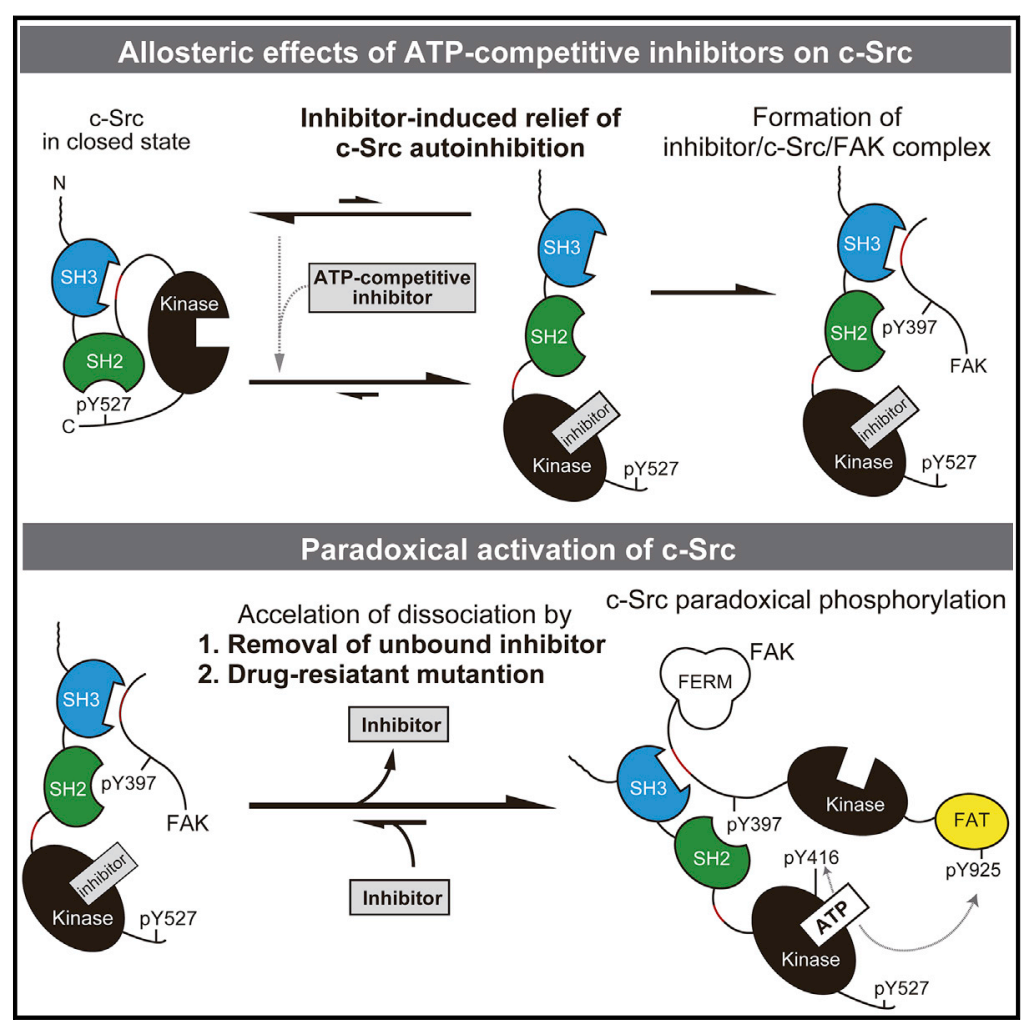

\section{Highlights}

- Binding of ATP-competitive inhibitors to c-Src relieves its auto-inhibition

- Inhibitor-bound c-Src in an open state forms a complex with FAK

- Reduction of inhibitor concentration activates c-Src and the FAK-Grb2-Erk cascade

- Inhibitors activate drug-resistant c-Src mutant and the cascade

\section{Authors}

Makio Higuchi, Kenichi Ishiyama, Masahiro Maruoka, Ryosuke Kanamori, Akifumi Takaori-Kondo, Naoki Watanabe

\section{Correspondence}

watanabe.naoki.4v@kyoto-u.ac.jp

\section{In brief}

Higuchi et al. report a molecular mechanism of drug-resistance. ATPcompetitive inhibitors directly turn c-Src into its active conformation, leading to its interaction with FAK. The reduction of inhibitor concentration or reduced inhibitor affinity for drug-resistant c-Src mutant enhances the dissociation of inhibitors from c-Src/FAK, leading to the paradoxical activation of C-Src-FAKGrb2-Erk signaling. 


\title{
Article \\ Paradoxical activation of c-Src as a drug-resistant mechanism
}

\author{
Makio Higuchi, ${ }^{1,5}$ Kenichi Ishiyama, ${ }^{1,2,6}$ Masahiro Maruoka, ${ }^{3,7}$ Ryosuke Kanamori, ${ }^{1}$ Akifumi Takaori-Kondo, ${ }^{2}$ \\ and Naoki Watanabe ${ }^{1,4,8, *}$ \\ ${ }^{1}$ Department of Pharmacology, Kyoto University Graduate School of Medicine, Kyoto, Japan \\ 2Department of Hematology and Oncology, Kyoto University Graduate School of Medicine, Kyoto, Japan \\ ${ }^{3}$ Laboratory of Single-Molecule Cell Biology, Tohoku University Graduate School of Life Sciences, Sendai, Japan \\ ${ }^{4}$ Laboratory of Single-Molecule Cell Biology, Kyoto University Graduate School of Biostudies, Kyoto, Japan \\ 5Present address: Medical Innovation Center, Kyoto University Graduate School of Medicine, Kyoto, Japan \\ ${ }^{6}$ Present address: Department of Microbiology and Immunology, University of California, San Francisco, San Francisco, CA, USA \\ ${ }^{7}$ Present address: Institute for Integrated Cell-Material Sciences, Kyoto University, Kyoto, Japan \\ 8Lead contact \\ *Correspondence: watanabe.naoki.4v@kyoto-u.ac.jp \\ https://doi.org/10.1016/j.celrep.2021.108876
}

\section{SUMMARY}

ATP-competitive inhibitors have been developed as promising anti-cancer agents. However, drug-resistance frequently occurs, and the underlying mechanisms are not fully understood. Here, we show that the activation of c-Src and its downstream phosphorylation cascade can be paradoxically induced by Src-targeted and RTK-targeted kinase inhibitors. We reveal that inhibitor binding induces a conformational change in c-Src, leading to the association of the active form c-Src with focal adhesion kinase (FAK). Reduction of the inhibitor concentration results in the dissociation of inhibitors from the c-Src-FAK complex, which allows c-Src to phosphorylate FAK and initiate FAK-Grb2-mediated Erk signaling. Furthermore, a drug-resistant mutation in c-Src, which reduces the affinity of inhibitors for c-Src, converts Src inhibitors into facilitators of cell proliferation by enhancing the phosphorylation of FAK and Erk in c-Src-mutated cells. Our data thus reveal paradoxical enhancement of cell growth evoked by target-based kinase inhibitors, providing potentially important clues for the future development of effective and safe cancer treatment.

\section{INTRODUCTION}

Selective ATP-competitive kinase inhibitors are becoming one of the mainstays of anti-cancer chemotherapy (Druker et al., 1996; Joseph et al., 2010). However, single-inhibitor treatments are often ineffective for suppressing target molecule signaling pathways, and to make matters worse, trigger the emergence of drug-resistant cancer cells, which in some cases have acquired drug-resistant mutations in the target molecule (Gorre et al., 2001). In other cases, cancer cells activate other kinases capable of reactivating the inhibitor-suppressed signaling pathway and reactivate survival and proliferation (Hirata et al., 2015; Johannessen et al., 2010).

The non-receptor tyrosine kinase c-Src, the product of the first described proto-oncogene, plays a critical role in mediating various signal transduction pathways by interacting with multiple molecules (Kim et al., 2009; Roskoski, 2015). c-Src contributes to cell survival and proliferation promoted by anchorage signaling that is triggered by an interaction between cell adhesion molecules and extracellular matrix (ECM) ligands (Cooper and Giancotti, 2019; Hamidi and Ivaska, 2018). In anchorage-dependent signaling, c-Src activates focal adhesion kinase (FAK), which is one of the crucial molecules for this signaling, leading to the downstream activation of Erk (Cooper and Giancotti, 2019; Hamidi and Ivaska, 2018). c-Src can also interact and cooperate with multiple tyrosine kinase receptors for growth factors, including epidermal growth factor (EGF), vascular endothelial growth factor (VEGF), platelet-derived growth factor (PDGF), hepatocyte growth factor (HGF), and insulin-like growth factor-1 (IGF-1). The aberrant activation of cSrc has been implicated in cancer progression with abnormal cell proliferation, apoptosis, angiogenesis, cell adhesion, migration, and invasion. Based on these notions, kinase inhibitor therapies targeting multiple tyrosine kinases including c-Src have been developed or are under development in clinical trials (Kim et al., 2009; Roskoski, 2015; Zhang and Yu, 2012).

Several reports have suggested that c-Src may contribute to drug resistance to kinase inhibitor therapy (Girotti et al., 2013, 2015; Hirata et al., 2015; Zhang et al., 2011). In tumor and cell lines that are treated with inhibitors, c-Src-enhanced activation of either adhesion signaling or c-Raf confers drug resistance and activation of Erk signaling (Girotti et al., 2015; Hirata et al., 2015), suggesting the possibility that c-Src may play an important role in drug resistance in cancer chemotherapy (Hirata et al., 2015; Johannessen et al., 2010). Thus, c-Src is one of the target molecules for anti-cancer kinase inhibitors, yet the precise mechanism by which c-Src confers drug resistance remains to be elucidated. 
A<smiles>COc1cc(Nc2c(C#N)cnc3cc(OCCCN4CCN(C)CC4)ccc23)c(Nc2cc(Nc3ncc(C(=O)Nc4c(C)cccc4Cl)s3)nc(C)n2)cc1Cl</smiles><smiles>CN1CCN(CCOc2cc3c(c(OC4CCOCC4)c2)C(Nc2c(Cl)ccc4c2OCO4)N=CN3)CC1</smiles><smiles>CCN(CC)CCc1ccc(-c2cc3c(NC(C)c4ccccc4)cccc3[nH]2)cc1</smiles><smiles>CC(C)c1ccc(-c2nn(C(C)(C)C)c3ncnc(N)c23)cc1</smiles><smiles>CC(C)(C)n1nc(-c2cccc3ccccc23)c2c(N)ncnc21</smiles><smiles>CC(C)(C)n1nc(Cc2cccc3ccccc23)c2c(N)ncnc21</smiles>

B
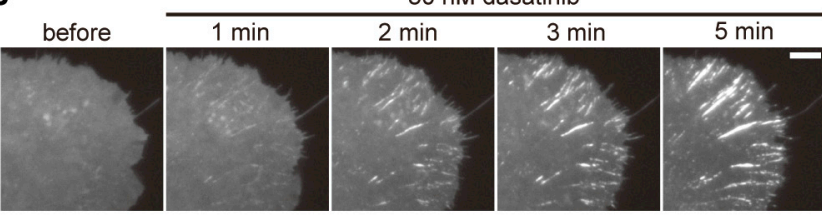

dasatinib
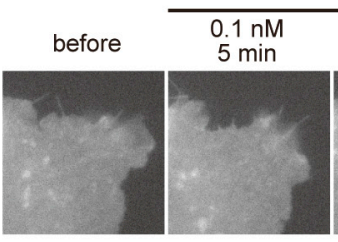

$1 \mathrm{nM}$
$5 \mathrm{~min}$

$10 \mathrm{nM}$
$5 \mathrm{~min}$
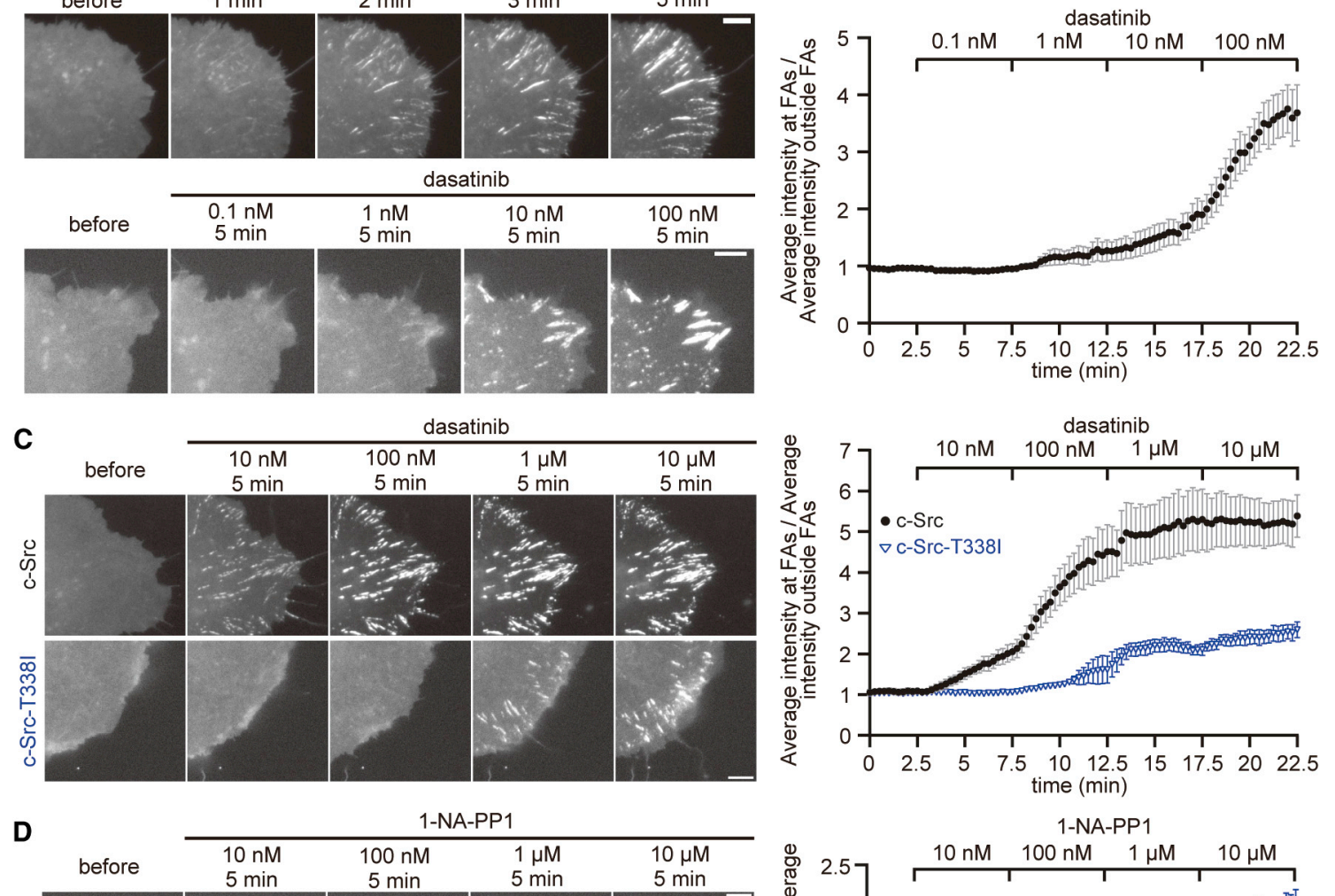

1-NA-PP1
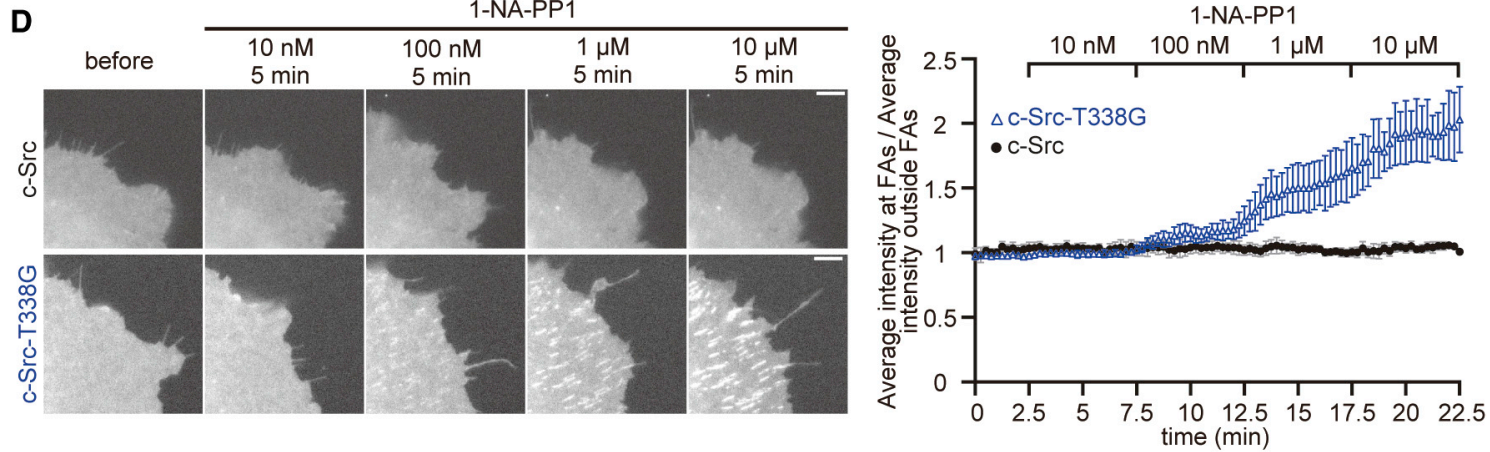

Figure 1. Kinase inhibitors induce translocation of c-Src to focal adhesions (FAs)

(A) Chemical structures of ATP-competitive inhibitors, dasatinib, bosutinib, saracatinib, PP2, NVP-AEE788, 1-NA-PP1, and 1-NM-PP1.

(B) Translocation of c-Src-EGFP to FAs induced by $30 \mathrm{nM}$ dasatinib (top) in XTC cells and its dose dependency (bottom). Drug concentrations were increased every $5 \mathrm{~min}$ (bottom). Scale bars, $5 \mu \mathrm{m}$. The right graph shows the ratio of c-Src-EGFP fluorescence intensity at FAs to that outside FAs. Data are presented as the means \pm SEMs $(n=3)$. See also Figures S1 and S2 and Video S1. 
In addition to a blockade of kinase activity, ATP-competitive inhibitors may change the conformation of target kinases, which sometimes induces hetero- or homo-dimerization and subcellular translocation in a manner that is similar to activation of the kinases (Hatzivassiliou et al., 2010; Okuzumi et al., 2009; Papa et al., 2003; Skora et al., 2013). In the case of Braf and IRE1, inhibitors may paradoxically activate target molecule signaling (Hatzivassiliou et al., 2010; Papa et al., 2003; Poulikakos et al., 2010). Our previous study has also suggested an allosteric effect of an ATP-competitive kinase inhibitor, imatinib, on Abelson tyrosine kinase (c-Abl) (Fujita et al., 2009). Imatinib induces the translocation of c-Abl to the lamellipodium tip (Fujita et al., 2009). Based on the domain requirement of imatinib-induced cell-edge translocation of c-Abl, we proposed that imatinib relieves the autoinhibition of c-Abl and enhances an interaction of C-Abl with partner proteins (Fujita et al., 2009). In this study, we concluded that the binding of imatinib converts C-Abl to an open, disassembled state and promotes association with its binding partner, abi-1/2 (Dai and Pendergast, 1995; Shi et al., 1995; Van Etten, 1999), which is responsible for the translocation of c-Abl (see below). Structural studies also demonstrated that $\mathrm{Abl}$ inhibitors disassemble the autoinhibited structure in c-Abl (Skora et al., 2013; Sonti et al., 2018). These examples highlight the possible modulation of target molecule signaling by the allosteric effects of kinase inhibitors apart from enzymatic inhibition.

In the present study, we discovered the allosteric effects of ATP-competitive inhibitors on the tyrosine kinase c-Src. c-Src is composed of $\mathrm{SH} 3, \mathrm{SH}$, tyrosine kinase domains, and a regulatory C-terminal. The $\mathrm{SH} 3$ and $\mathrm{SH} 2$ domains bind to the prolinerich region and phosphorylated Tyr-527 in c-Src, respectively, which maintains the closed state of c-Src (Figure S1A; Xu et al., 1997; Yeatman, 2004). The dephosphorylation of Tyr-527 relieves the autoinhibition of $\mathrm{c}-\mathrm{Src}$, leading to the interaction of c-Src with partner molecules via its $\mathrm{SH} 3$ and $\mathrm{SH} 2$ domains (Figure S1A; Yeatman, 2004). We found that binding ATP-competitive inhibitors to the $\mathrm{c}$-Src ATP pocket also relieves autoinhibition in $\mathrm{c}-\mathrm{Src}$ and induces the association of $\mathrm{c}-\mathrm{Src}$ and FAK both in cells and in vitro. Although inhibitors should abrogate the c-Src kinase activity, we found that washout of low-affinity Src inhibitors, but not high-affinity inhibitors, can promote the phosphorylation of FAK pre-complexed with $\mathrm{c}$-Src in the presence of inhibitors. Importantly, a drug-resistant mutation in c-Src, which reduces the affinity to inhibitors, was found to convert even highaffinity Src inhibitors to the facilitators of Src-FAK-Grb2-Erk signaling. In the presence of the drug-resistant Src mutant, inhibitors promoted the phosphorylation of FAK without washout, leading to enhanced cell proliferation. Our data thus reveal that an inhibitor-bound kinase may have a high propensity to form a complex with its substrate protein, which may lead to the activation of signaling when inhibitors do not bind the kinase tightly. If cells acquire a drug-resistant mutation, then inhibitors may propagate a cancer-promoting signal. Our findings prompt reconsideration of the basic strategy behind the development of kinase inhibitor therapies.

\section{RESULTS}

Inhibitors directly relieve autoinhibition of c-Src, leading to ligand binding of $\mathrm{SH} 3$ and $\mathrm{SH} 2$ domains Using high-resolution live-cell imaging, previously we observed that imatinib induces leading-edge translocation of c-Abl, which suggested that imatinib relieves an autoinhibitory interaction in $\mathrm{c}-\mathrm{Abl}$ and promotes the binding of $\mathrm{c}-\mathrm{Abl}$ to partner molecules (Fujita et al., 2009). We therefore investigated the effects of kinase inhibitors on the intracellular localization of c-Src using a similar approach.

Four distinct Src inhibitors, dasatinib (O'Hare et al., 2005), bosutinib (Boschelli et al., 2001), saracatinib (Green et al., 2009), and PP2 (Bain et al., 2007) and a receptor tyrosine kinase inhibitor NVP-AEE788 (AEE788) (Traxler et al., 2004) induced the rapid translocation of $\mathrm{C}$-Src-EGFP to focal adhesions (FAs) marked by TagRFP-T-FAK in Xenopus laevis XTC cells (Figures 1A, 1B, S1B, S1C, and S2D; Table S1; Video S1). These drugs induced the translocation of $\mathrm{C}-\mathrm{Src}$ in a dose-dependent manner (Figures 1B, S1B, S1C, and S2D; Table S1; Video S1). In unstimulated cells, c-Src-EGFP localizes mainly on the plasma membrane and only scarcely associates with FAs (Figures 1B and $\mathrm{S1C})$. The inhibitor-induced localization of $\mathrm{C}-\mathrm{Src}$ was similar to the localization of c-Src-K249E-P250E-Y527F (Figures 1B, 2A, and $S 1 A$ ), which is a constitutively active kinase with an open conformation due to defects in autoinhibition elicited by the proline-rich region and tyrosine phosphorylation at Y527 (Gonfloni et al., 1999; Krishnamurty et al., 2013).

A drug-resistant gatekeeper mutant C-Src-T338I, which has reduced affinities to inhibitors (Apsel et al., 2008; Azam et al., 2008; Levinson and Boxer, 2014), did not translocate to FAs at concentrations at which the translocation of wild-type c-Src was effectively induced (Figures 1C, S2A, and S2D; and Video S2). At higher concentrations, C-Src-T338I-EGFP weakly associated with FAs (Figures 1C, S2A, S2D; Video S2). Compared with wild-type, 1-2 orders of magnitude higher concentrations of kinase inhibitors were required to induce the translocation of $\mathrm{c}$ Src-T338I (Figures 1C, S2A, and S2D; Video S2). These data support the idea that drug binding to $\mathrm{C}$-Src induces the translocation of c-Src to FAs. To further exclude the possibility that the effect of inhibitors arises from the inhibition of kinases other than c-Src, we tested the combination of an analog-sensitive c-SrcT338G mutant with PP1 analogs selective to C-Src-T338G (Bishop et al., 2000). The analog-sensitive c-Src-T338G mutant can be selectively inhibited by analog-sensitive inhibitors 1NA-PP1 and 1-NM-PP1 (Figure 1A), which only weakly inhibit wild-type c-Src (in vitro half-maximal inhibitory concentration

(C) Live-cell imaging of drug-resistant mutant c-Src-T338I-EGFP (top) or c-Src-EGFP (bottom) treated with different concentrations of dasatinib in XTC cells. Drug concentrations were increased every $5 \mathrm{~min}$. Scale bars, $5 \mu \mathrm{m}$. The graph shows the ratio of c-Src-T338I-EGFP or c-Src-EGFP fluorescence intensity at FAs to that outside FAs. Data are presented as the means \pm SEMs $(n=3)$. See also Figure $S 2$.

(D) Translocation of an analog-sensitive mutant c-Src-T338G-EGFP (top), but not c-Src-EGFP (bottom), upon treatment with a PP1 analog 1-NA-PP1 in XTC cells. Drug concentrations were increased every $5 \mathrm{~min}$. Scale bars, $5 \mu \mathrm{m}$. The graph shows the ratio of fluorescence intensity at FAs to that outside FAs. Data are presented as the means \pm SEMs $(n=3)$. See also Figure S2. 

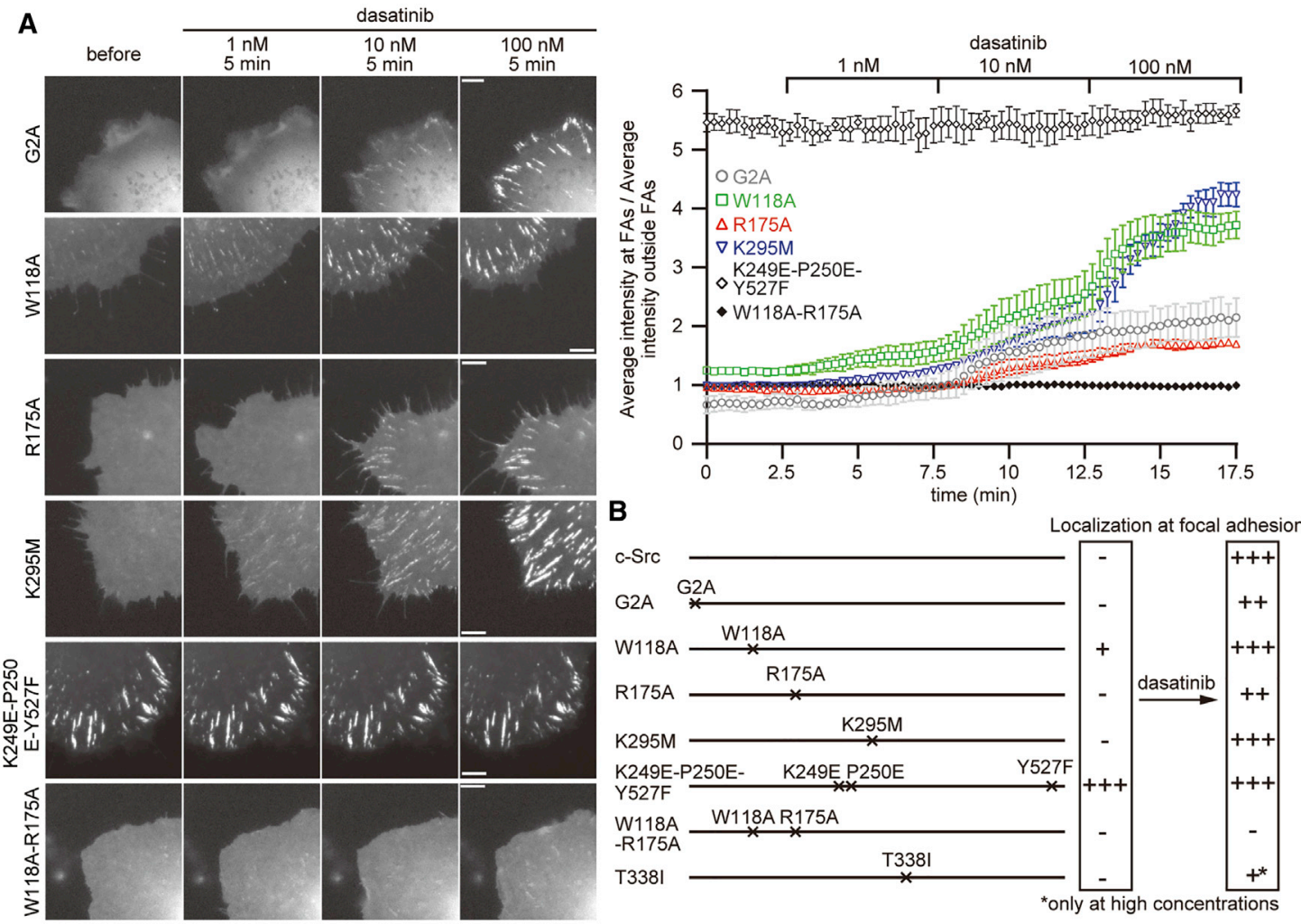

Figure 2. Kinase inhibitors relieve autoinhibition in c-Src, leading to ligand binding of SH3 and SH2 domains

(A) Response of C-Src-EGFP mutants G2A, W118A, R175A, K295M, K249E-P250E-Y527E, and W118A-R175A in XTC cells. Drug concentrations were increased every $5 \mathrm{~min}$. Scale bars, $5 \mu \mathrm{m}$. The graph shows the ratio of the fluorescence intensity at FAs to that outside FAs. Data are presented as the means $\pm S E M s(n=3)$. See also Figure S2 and Video S3.

(B) Summary of the localization of c-Src-EGFP mutants before and after treatment with inhibitors. "-" indicates that mutants are not associated with FAs. "+" indicates that the intensity at FAs is marginally stronger than outside FAs. "++" indicates that the mutant clearly localizes both at FAs and outside FAs. "+++" indicates that the majority of the mutant protein localizes at FAs. See also Figure S2.

[ $\left[\mathrm{C}_{50}\right], 0.9$ and $1.2 \mu \mathrm{M}$, respectively) but strongly inhibit the analog-sensitive c-Src-T338G mutant (in vitro $\mathrm{IC}_{50}, 1.5$ and $2 \mathrm{nM}$, respectively) (Table S1; Zhang et al., 2013). 1-NA-PP1 and 1-NM-PP1 induced the translocation of c-Src-T338GEGFP to FAs in a dose-dependent manner, whereas these analogs did not enhance the localization of wild-type c-Src-EGFP (Figures 1D, S2B, and S2D; Video S2). These results suggest that the binding of inhibitors to the ATP-binding pocket of cSrc triggers its translocation to FAs.

To explore which structures in c-Src are required for the inhibitor-induced translocation of C-Src to FAs, we examined the subcellular localization of various Src mutants before and after treatment with dasatinib, bosutinib, saracatinib, PP2, and AEE788. A G2A mutation that abrogates N-myristoylation (Bagrodia et al., 1993; Patwardhan and Resh, 2010) mobilized c-Src to the cytoplasm without impairing inhibitor-induced translocation to FAs (Figures 2A, S2C, and S2D; Video S3). A kinasedefective K295M (Okamura and Resh, 1995) mutant localized at the plasma membrane and translocated to FAs in response to the inhibitors (Figures 2A, S2C, and S2D; Video S3), indicating that the inhibition of the kinase activity of c-Src is not a trigger of translocation. Next, we introduced point mutations in c-Src regulatory domains, which impair either autoinhibitory intramolecular interactions or the ligand binding of c-Src. A W118A mutant, with impaired ligand-binding abilities of the $\mathrm{SH} 3$ domain (Espada and Martín-Pérez, 2017; Fincham et al., 2000; Liu et al., 1993), weakly localized at FAs in the absence of the inhibitors, and the inhibitors enhanced the localization of W118A at FAs (Figures 2A, S2C, and S2D; Video S3). In contrast, an SH2-deficient mutant, R175A, devoid of binding abilities for tyrosinephosphorylated ligands (Brunton et al., 2005; Gottlieb-Abraham et al., 2013; Verderame, 1997) did not localize at FAs before treatment (Figures 2A, S2C, and S2D; Video S3). The inhibitors weakly enhanced the localization of R175A c-Src at FAs to a lesser degree than wild-type c-Src (Figures 2A, S2C, and S2D; Video S3). The constitutively active K249E-P250E-Y527F mutant showed prominent localization at FAs, which was scarcely enhanced by the inhibitors (Figures 2A, S2C, and S2D; Video S3). A Src mutant harboring dual W118A and R175A mutations stayed on the plasma membrane in the absence or presence of the inhibitors (Figures 2A, S2C, and S2D; Video S3).

Thus, the simultaneous impairment of $\mathrm{SH} 3$ and $\mathrm{SH} 2$ domains abrogates the inhibitor-induced translocation of c-Src to FAs, whereas defects in each domain partially impair the 
A
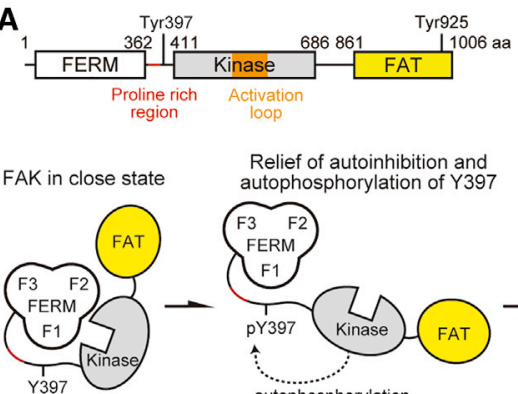
Relief of autoinhibition and
autophosphorylation of $Y 397$ F3 F2

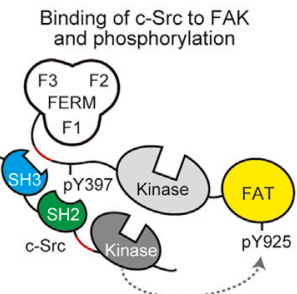

B

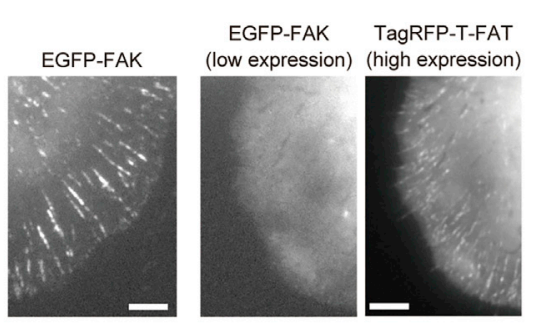

C

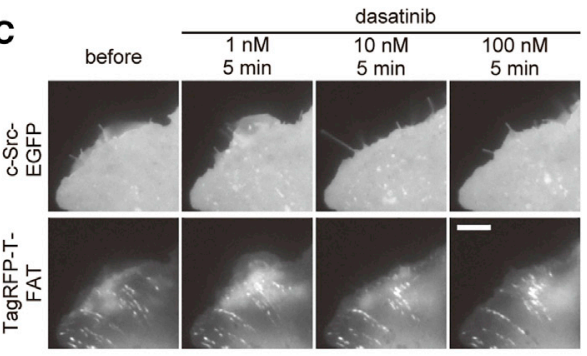

D
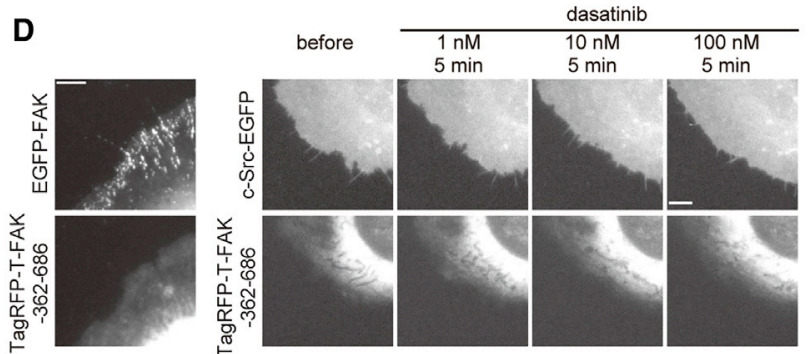

E

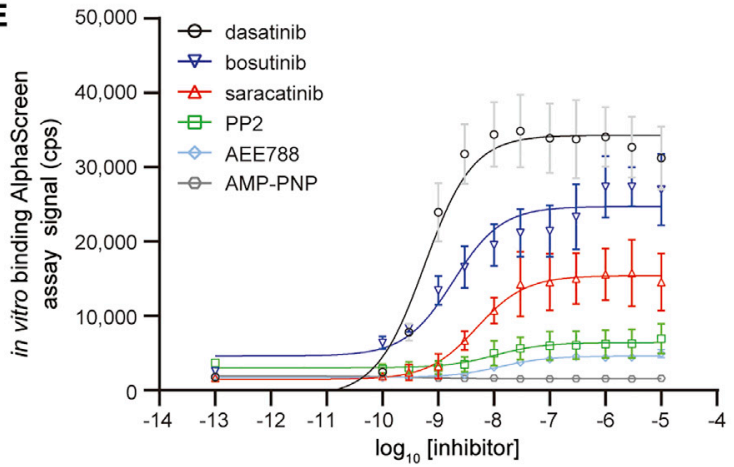

$\mathbf{F}$
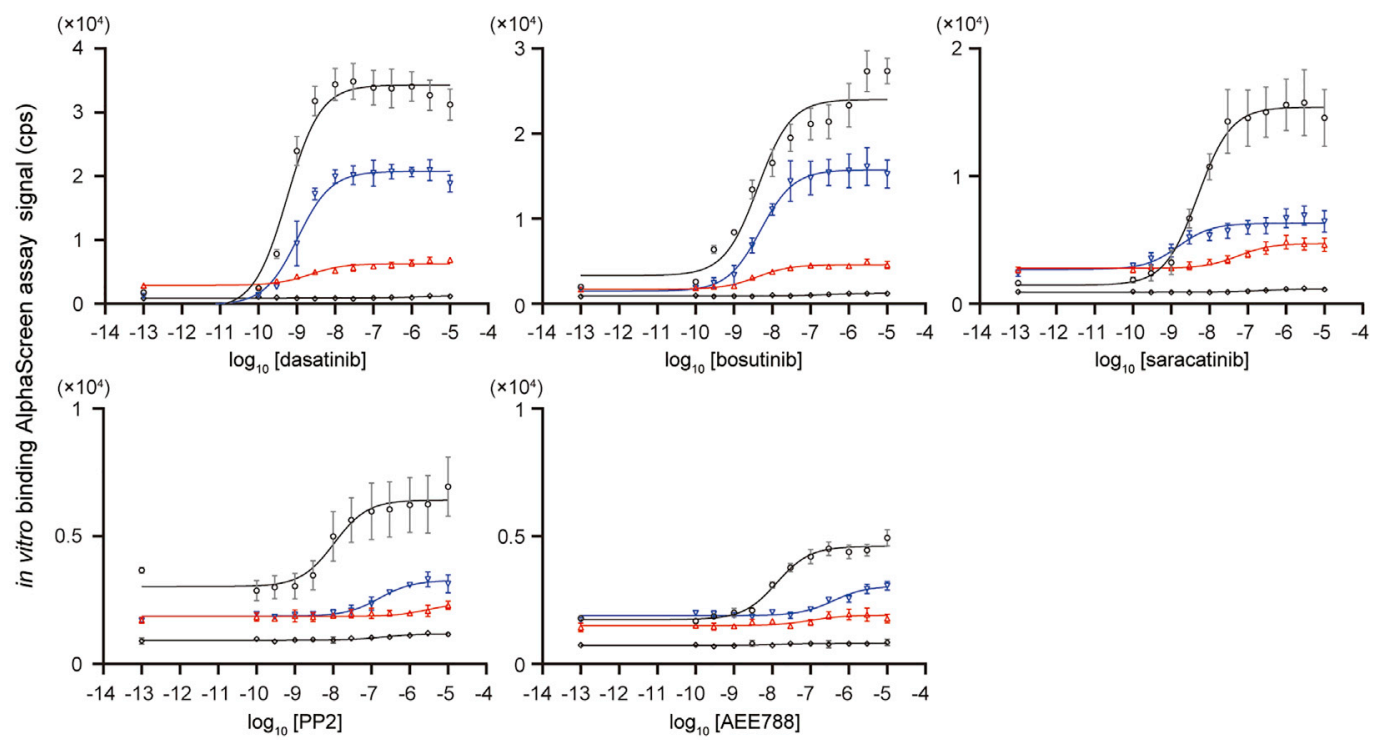

$\log _{10}[$ AEE 788$]$ 
translocation (Figure 2B). These data indicate that the inhibitor binding to the kinase domain allosterically disassembles c-Src to an open state, thereby promoting the interaction of c-Src $\mathrm{SH} 2$ and $\mathrm{SH} 3$ domains with a partner molecule(s) at FAs.

\section{Kinase inhibitor-bound c-Src binds to FAK}

The localization of c-Src at FAs depends on FAK (Yeo et al., 2006). FAK consists of FERM (Band 4.1, Ezrin, Radixin, and Moesin), tyrosine kinase, and FAT (focal adhesion targeting) domains (Figure 3A; Sulzmaier et al., 2014). A linker between the FERM and kinase domains contains a proline-rich region and a tyrosine autophosphorylation site at amino acid (aa) 397 (Figure 3A; Lietha et al., 2007; Sulzmaier et al., 2014). An interaction of the FERM domain with the kinase domain inhibits kinase activity, and lipid binding to the FERM domain relieves autoinhibition, leading to autophosphorylation at tyrosine 397 (Lietha et al., 2007; Sulzmaier et al., 2014). The phosphorylated linker region binds to partner molecules through $\mathrm{SH} 3$ and $\mathrm{SH} 2$ domains of the partners (Sulzmaier et al., 2014). The FAT domain is responsible for the localization of FAK at FAs (Hildebrand et al., 1993; Koshman et al., 2010). We therefore tested whether inhibitorinduced translocation of $\mathrm{c}$-Src requires FAK.

Overexpression of the FAT domain of FAK is known to abrogate the localization of FAK at FAs (Koshman et al., 2010). We confirmed that the overexpression of the FAT domain, which localized at FAs, abolished the localization of full-length FAK at FAs in XTC cells (Figure 3B). Dasatinib did not induce the translocation of c-Src-EGFP in cells overexpressing TagRFP-T-FAT (Figure $3 \mathrm{C}$ ). We also tested the effect of overexpression of a FAK mutant, FAK-362-686, which contains Src-interacting motifs (Figure $3 A$ ) but lacks both FERM and FAT domains. FAK362-686 localized in the cytoplasm and had no effect on the localization of full-length FAK at FAs (Figure 3D). In addition, the overexpression of FAK-362-686 suppressed the dasatinibinduced translocation of c-Src-EGFP to FAs (Figure 3D), which is attributable to the sequestration of inhibitor-bound $\mathrm{c}-\mathrm{Src}$ by FAK-362-686 overexpressed in the cytoplasm. These results indicate that inhibitor-induced translocation of $\mathrm{c}$-Src requires the interaction of $\mathrm{c}-\mathrm{Src}$ with FAK localized at FAs.
We next examined whether kinase inhibitors directly enhance the binding of c-Src to FAK. We purified c-Src and GST (glutathione S-transferase)-pY397-FAK ${ }^{362-411}$, which make up the binding site for the $\mathrm{c}-\mathrm{Src} \mathrm{SH} 2$ and $\mathrm{SH} 3$ domains, and performed in vitro binding analysis using the AlphaScreen assay. The binding of c-Src to pY397-FAK ${ }^{362-411}$ was enhanced with increasing doses of kinase inhibitors, and the half-maximal effective concentration $\left(E_{50}\right)$ values of inhibitors for enhancing the binding of c-Src to FAK were close to the $\mathrm{IC}_{50}$ for $\mathrm{c}$-Src kinase activities (Figure 3E; Table S1). These inhibitors also enhanced the binding of $c-S r c$ to proline-rich deficient pY397-P371A-P374AFAK $^{362-411}$ (Heim et al., 2017), although binding signals were lower than those with pY397-FAK ${ }^{362-411}$ (Figure 3F), implying a contribution of the $\mathrm{C}-\mathrm{Src} \mathrm{SH} 3$ domain to FAK binding. Compared to pY397-FAK ${ }^{362-411}$ and pY397-P371A-P374A-FAK ${ }^{362-411}$, unphosphorylated GST-Y397-FAK ${ }^{362-411}$ bound inhibitor-treated c-Src only weakly (Figure $3 \mathrm{E}$ ), indicating that binding of the cSrc SH2 domain to pY397 in FAK plays a major role in the inhibitor-induced binding of c-Src to FAK. A non-hydrolyzable ATP analog, AMP-PNP, did not enhance the binding (Figure 3D). These data indicate that the binding of ATP-competitive inhibitors, but not an ATP analog, of the c-Src ATP-binding site relieve autoinhibitory interactions in c-Src (i.e., $\mathrm{SH} 3$ and $\mathrm{SH} 2$ domains to the proline-rich motif and C-terminal phosphotyrosine, respectively), leading to the binding of the $\mathrm{c}-\mathrm{Src} \mathrm{SH} 3$ and $\mathrm{SH} 2$ domains to FAK.

In addition, we verified the binding partner of imatinib-bound c-Abl using a similar approach. We previously proposed that an $\mathrm{Abl}$ inhibitor, imatinib, relieves the autoinhibition of c-Abl, leading to the binding of c-Abl with unidentified partner molecules at the lamellipodium tip (Fujita et al., 2009). Abi1/2 was a strong candidate because Abi1/2 binds the $\mathrm{SH} 3$ domain and the proline-rich motif of c-Abl (Dai and Pendergast, 1995; Shi et al., 1995; Van Etten, 1999), both of which contribute to the imatinib-induced cell edge translocation of c-Abl (Figure S3A; Fujita et al., 2009). Moreover, Abi-1 localizes at the lamellipodium tip (Figure S3B) as a part of the WAVE complex (Nakagawa et al., 2003; Millius et al., 2012). We therefore tested whether Abi-1 is responsible for imatinib-induced translocation of c-Abl.

Figure 3. Kinase inhibitor-bound c-Src binds to FAK

(A) The structure of FAK and a schematic of the interaction with c-Src. FAK consists of FERM, kinase, and FA targeting (FAT) domains (top). The interaction between FERM and the kinase domains maintains autoinhibition in FAK. Binding of the FERM domain to lipids leads to the relief of autoinhibition, and the kinase domain autophosphorylates Tyr397 in the linker between FERM and the kinase domains. The proline-rich region and pTyr397 bind SH3 and SH2 domains of c-Src in an open state, leading to the phosphorylation of Tyr925 in the FAT domain. Phosphorylated Tyr925 binds to other molecules such as Grb2, leading to the activation of Ras-Erk signaling. The FAT domain is required for the localization of FAK at FAs.

(B) EGFP-FAK localized at FAs in XTC cells (left). Overexpression of the TagRFP-T-tagged FAT domain, which localized at FAs, abolished localization of EGFPFAK at FAs (center and right). Scale bars, $5 \mu \mathrm{m}$. See also Figure $S 3$.

(C) Impaired dasatinib-induced translocation of c-Src-EGFP to FAs in XTC cells overexpressing TagRFP-T-FAT. The concentration of dasatinib was increased every 5 min. Scale bar, $5 \mu \mathrm{m}$.

(D) FERM and FAT domain-deleted FAK mutant (FAK-362-686), which make up the Src-interacting motifs, localized not at FAs but in the cytoplasm, and had no effect on the localization of FAK at FAs in XTC cells (left). The overexpression of FAK-362-686 abolished the dasatinib-induced translocation of c-Src-EGFP to FAs (right).

(E) In vitro binding analysis of purified recombinant $6 \times$ His-tagged c-Src and glutathione S-transferase (GST)-tagged phosphorylated Y397 FAK ${ }^{362-411}$ treated with inhibitors. To generate GST-pY397 FAK ${ }^{362-411}$, GST-FAK ${ }^{362-411}$ was phosphorylated by purified recombinant FAK kinase (FAK ${ }^{411-686}$ ) (see also Method details). The binding assay was monitored by the AlphaScreen assay. Data are presented as the means $\pm \mathrm{SEMs}\left(\mathrm{n}=3\right.$ ). $\mathrm{EC}_{50}$ values for dasatinib, bosutinib, saracatinib, PP2, and NVP-AEE788 (AEE788) were 0.56, 1.9, 5.0, 106.2, and $129.7 \mathrm{nM}$, respectively.

(F) AlphaScreen-based binding assay of $6 \times$ His-tagged c-Src with GST-tagged phosphorylated Y397 FAK ${ }^{362-411}$, GST-tagged phosphorylated Y397-P371AP374A-FAK ${ }^{362-411}$, GST-tagged non-phosphorylated Y397 FAK ${ }^{362-411}$, or GST. Samples were treated with dasatinib, bosutinib, saracatinib, PP2, or AEE788. Data are presented as the means \pm SEMs $(n=3)$. 
c-Abl is distributed throughout the entire lamellipodia through its C-terminal F-actin-binding motif, and imatinib induced the translocation of c-Abl to the lamellipodium tip, where Abi-1 was highly accumulated (Figure S3B). The overexpression of Abi-1 fused with the mitochondria-targeting motif of ActA (Bear et al., 2000) recruited c-Abl to mitochondria upon treatment with imatinib (Figure S3C). Overexpression of WAVE-mito, which relocalized Abi-1 from the cell edge to mitochondria abrogated the translocation of c-Abl to the lamellipodium tip (Figure S3D). We further tested whether imatinib enhances the binding of $c-A b l$ to Abi-1 in an in vitro binding assay. Imatinib enhanced the binding in a dose-dependent manner, and the $\mathrm{EC}_{50}$ value of imatinib in the in vitro binding assay (Figure S3E) was similar to the $\mathrm{IC}_{50}$ of imatinib for $c-A b l$ kinase activities $\left(K_{d}\right.$ value of imatinib to $c-A b l$, $37 \mathrm{nM}$ ) (Schindler et al., 2000). Our data indicate that ATPcompetitive inhibitor-induced relief of autoinhibition in target kinases and subsequent binding to partner molecules are not specific for c-Src.

Reduction of kinase inhibitors paradoxically activates cSrc and FAK-Grb2-Erk signaling

The data presented above suggest that ATP-competitive inhibitors may work as allosteric facilitators of c-Src conformational activation, leading to the formation of a c-Src/FAK complex. We therefore hypothesized that decreasing drug concentration, which may result in the dissociation of inhibitors from the c-Src/ FAK complex, allows c-Src to phosphorylate FAK. To test this, we performed an in vitro kinase assay. In the presence of low-affinity inhibitors for c-Src, PP2, and AEE788, autophosphorylation of Y416 and phosphorylation of Y925 in FAK were suppressed as compared with DMSO-treated samples (Figure 4A). After washout of the drugs, inhibitor-treated $\mathrm{C}-\mathrm{Src}$ remained bound to FAK, and both c-Src (Y416) and FAK (Y925) were phosphorylated upon the addition of ATP, indicating that $\mathrm{C}-\mathrm{Src}$ and FAK can be activated by the addition and washout of the drugs (Figure 4A). High-affinity Src inhibitors dasatinib, bosutinib, and saracatinib also suppressed phosphorylation of both c-Src and FAK (Figure S4A). After washout of these inhibitors, c-Src still strongly bound to FAK. However, phosphorylation of FAK did not increase upon washout of the high-affinity inhibitors (Figure S4A).

Phosphorylated $\mathrm{Y} 925$ of FAK interacts with the $\mathrm{SH} 2$ domain of Grb2, leading to the activation of Erk signaling (Schlaepfer et al., 1994). We confirmed that Grb2-EGFP localized at FAs in XTC cells, and the localization of Grb2-EGFP was rapidly enhanced by the imidazole-induced artificial activation of c-Src-R388AY527F (Figures 4B and S4B), the catalytic activity of which can be rescued by imidazole (Qiao et al., 2006; Oikawa et al., 2008). In contrast, Grb2-EGFP localization at FAs was diminished by Src inhibitors (Figures $4 \mathrm{C}$ and S4C). We therefore used Grb2-EGFP localization as an indicator of c-Src activation at FAs in live-cell imaging.

Treatment with low-affinity Src inhibitors PP2 and AEE788 diminished the localization of Grb2-EGFP at FAs (Figure 4C; Video S4). Subsequent washout enhanced Grb2-EGFP signals at FAs (Figure 4C; Video S4). Grb2-EGFP signals after drug washout were stronger than before the treatment with inhibitors (Figure 4C; Video S4). DMSO treatment and subsequent washout did not enhance Grb2-EGFP signals at any time point
(Figure 4C; Video S4). Furthermore, this inhibitor washoutinduced translocation of Grb2-EGFP was dependent on the localization of FAK at FAs (Figure S4D). High-affinity Src inhibitors dasatinib, bosutinib, and saracatinib also suppressed Grb2 signals at FAs (Figure S4C; Video S4). However, washout did not recover Grb2 signals compared with those before stimulation (Figure S4C; Video S4), suggesting that these high-affinity Src inhibitors (Table S1) may still bind to the c-Src/FAK complex after washout.

We also examined the effects of inhibitor treatment and subsequent washout in human breast cancer cell line MCF-7; note that T338, Y416, and Y527 in chicken c-Src correspond to T341, Y419, and Y530 in human c-Src, respectively. Treatment with Src inhibitors and the RTK inhibitor did not enhance the activities of C-Src, FAK, and Erk (Figures 4D and S4E). However, the washout of PP2 and AEE788 enhanced the activities of c-Src, FAK, and Erk, as judged by the phosphorylation of c-Src (Y419), FAK (Y925), and Erk (T202/Y204) (Figure 4D). Their activation after drug washout did not occur in cells treated with DMSO, dasatinib, bosutinib, or saracatinib (Figures 4D and S4E).

Thus, the association and subsequent dissociation of inhibitors from c-Src may induce the unexpected paradoxical activation of $\mathrm{c}$-Src, leading to the activation of the FAK-Erk signaling pathway. Our data show that inhibitor washout-induced activation of c-Src is evoked only by low-affinity inhibitors for c-Src, suggesting that fast dissociation of inhibitors from the c-Src/ FAK complex may be a prerequisite for the paradoxical activation of c-Src.

Kinase inhibitors activate a drug-resistant c-Src mutant, leading to Erk activation

Mutations at the gatekeeper residue Thr338 (human number 341) in chicken c-Src (e.g., c-Src-T338I) cause resistance to multiple kinase inhibitors by lowering the affinity for the inhibitors (Table S1; Apsel et al., 2008; Azam et al., 2008; Levinson and Boxer, 2014). We hypothesized that such a reduced affinity for inhibitors may give rise to the rapid dissociation of inhibitors from the inhibitor-induced c-Src-FAK complex, thereby potentiating c-SrcFAK signaling. In an in vitro kinase assay, multiple inhibitors, including dasatinib, bosutinib, saracatinib, PP2, and AEE788 enhanced the phosphorylation of Y416 in C-Src-T338I and the phosphorylation of Y925 in FAK (Figure 5A). This enhanced phosphorylation was not accompanied by the dephosphorylation of Y527, whereas Src-Y527F, a constitutively active c-Src with an open conformation, efficiently phosphorylated FAK (Figure 5A). These results are consistent with the idea that inhibitors convert C-Src-T338I into an open configuration to allow its binding to FAK, hence the formation of the inhibitor-bound C-Src-T338I/ FAK complex. We also confirmed that inhibitors enhance the binding of drug-resistant c-Src-T338I to FAK in vitro. Inhibitors enhanced the binding of C-Src-T338I to FAK at higher concentrations (Figure 5B) than wild-type c-Src in vitro (Figure 3C), suggesting that inhibitors relieve autoinhibition in c-Src-T338I, leading to the binding of c-Src-T338I and FAK. Because of the reduced drug affinity for the drug-resistant c-Src mutant, inhibitors are expected to dissociate faster from c-Src-T338I than wild-type cSrc. The data (Figure 5A) indicate that inhibitors may dissociate from the complex while retaining the active open configuration 
A
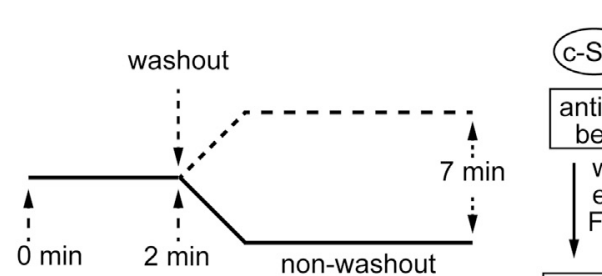

in

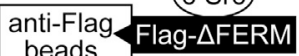

washout to remove FAK-unbound c-Src

c-Src

anti-Flag
beads
extra inhibitors and

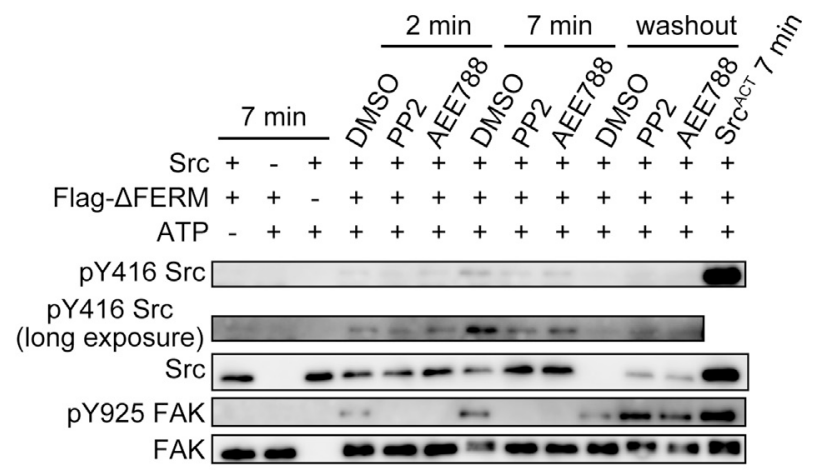

D

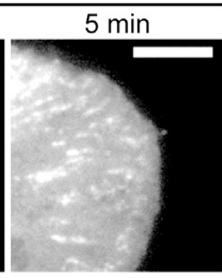

pY419 Src

after

after

c before drug treatment $5 \mathrm{~min}$ washout

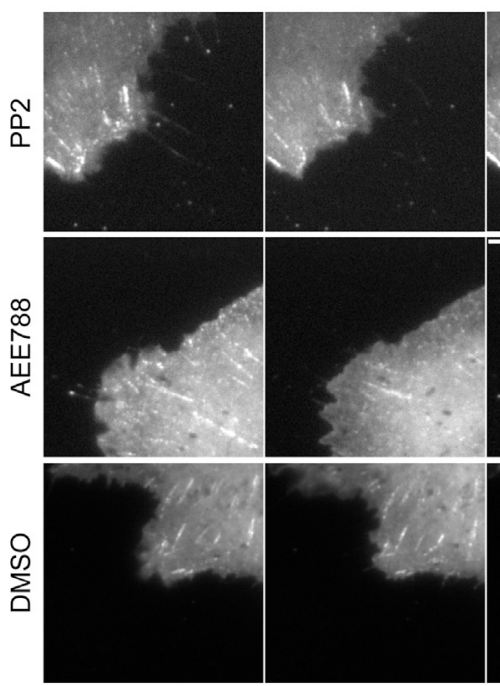

Src

pY925 FAK

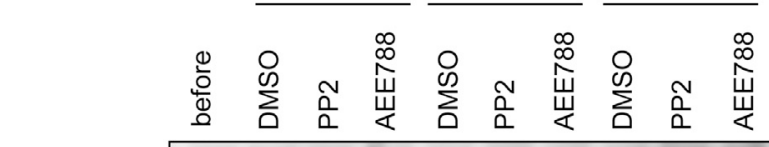

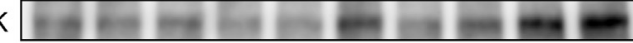
FAK

pErk

Erk
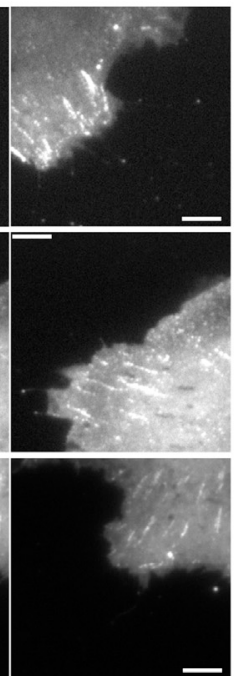

Figure 4. Washout of kinase inhibitors paradoxically activates c-Src, leading to activation of FAK-Grb2-mediated Erk signaling

(A) Western blot analysis of phospho-Y416 Src, Src (detected with a rabbit polyclonal antibody), pY925 FAK, and FAK in an in vitro kinase assay. C-Src and FLAGFAK $\Delta$ FERM-bound beads were treated with inhibitors. In lanes indicated by "washout," after treatment with $0.1 \%$ DMSO, $10 \mu$ PP2, or $10 \mu M$ NVP-AEE788 (AEE788) with 2 mM ATP for 2 min, washout was carried out and samples were then incubated for 5 min after the addition of 2 mM ATP. Src ACT indicates C-SrcK249E-P250E-Y527F. See also Figure S4.

(B) Enhanced localization of Grb2-EGFP at FAs induced by $10 \mathrm{mM}$ imidazole in XTC cells expressing c-Src-R388A-Y527F-TagRFP-T (8A7F-TagRFP-T). Scale bar, $5 \mu \mathrm{m}$.

(C) Live-cell imaging of Grb2-EGFP with treatment and subsequent washout of low-affinity inhibitors in XTC cells. PP2 (10 $\mu M)$, NVP-AEE788 (AEE788) (10 $\mu$ M), or $0.1 \%$ DMSO alone were used. The treatment was performed for $5 \mathrm{~min}$. Images before, $5 \mathrm{~min}$ after drug treatment, and 10 min after washout are shown. Scale bars, $5 \mu \mathrm{m}$. See also Figure S4 and Video S4.

(D) Western blot analysis of phospho-Y419 Src, Src (detected with a mouse monoclonal antibody), phospho-Erk (pErk), and Erk1\&2 (Erk) in MCF-7 cells treated with inhibitors and subjected to inhibitor washout. In lanes indicated by washout, after treatment with $0.1 \%$ DMSO, $10 \mu \mathrm{M}$ PP2, or $10 \mu \mathrm{M}$ NVP-AEE788 (AEE788) for $10 \mathrm{~min}$, inhibitor washout was carried out and samples were further incubated for 20 min. See also Figure S4. 
A

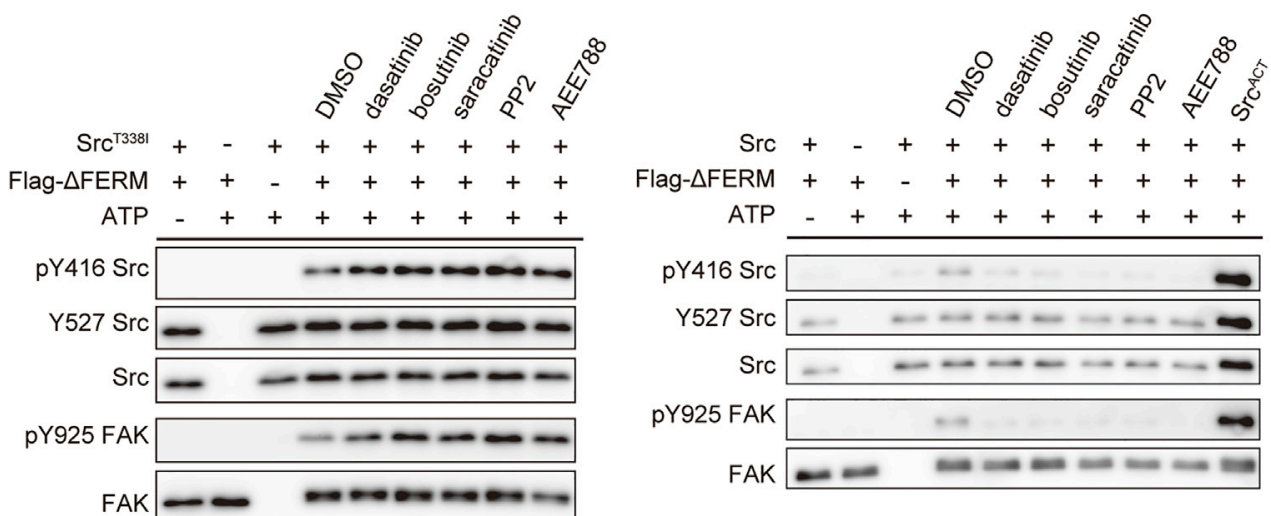

B

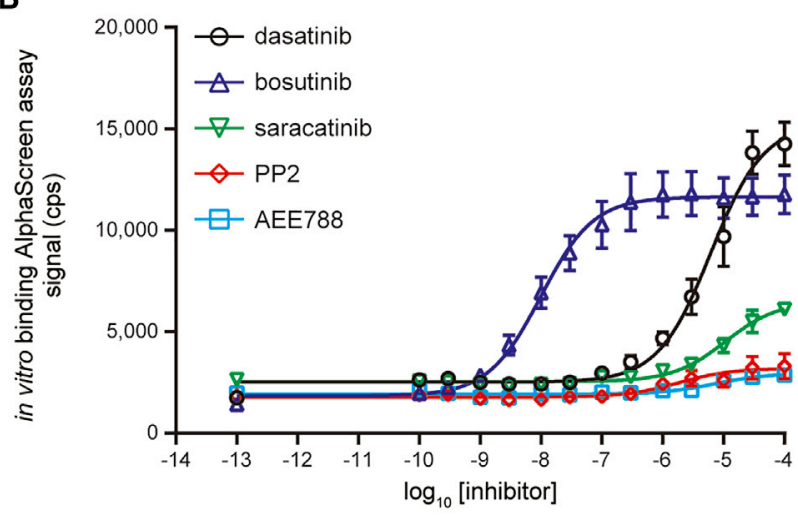

C

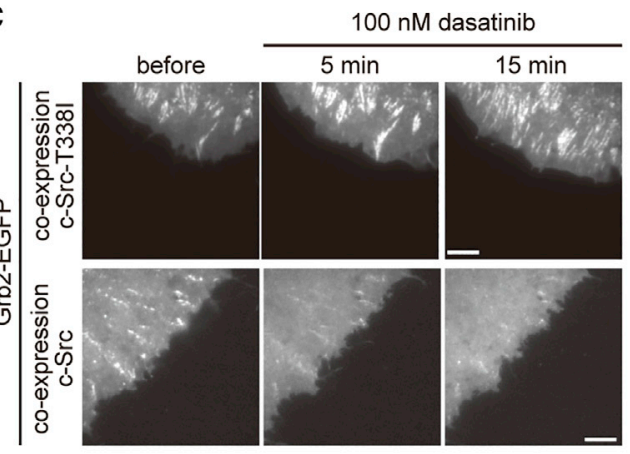

D

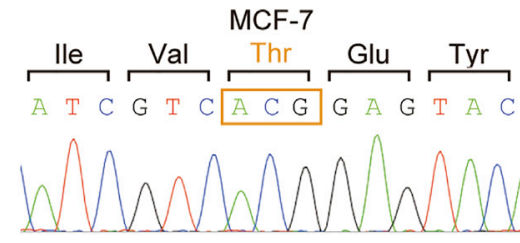

MCF-7-T341I

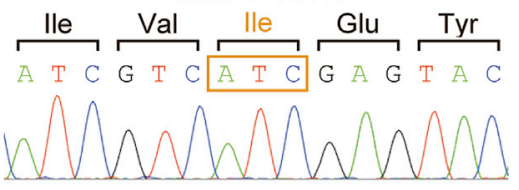

E

MCF-7-T341I

MCF-7

dasatinib $(\mu \mathrm{M})$ $\frac{\text { dasatinib }(\mu \mathrm{M})}{\stackrel{0}{0}}$

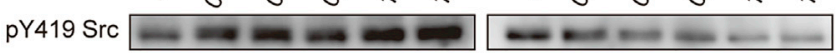

Y530 Src - — -

Src

- - - - -

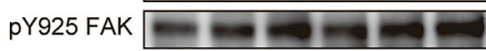

$\mathrm{FAK}=-\cdots$

pErk $\because \cdots \cdots=$

Erk $= \pm \equiv=$

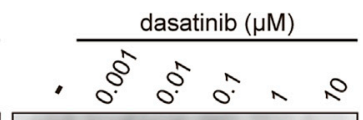

$-\cdots+\cdots$
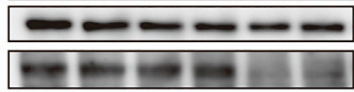

$-\infty-\infty$
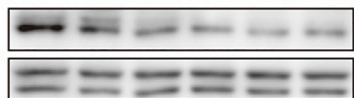

$\mathbf{F}$

$\nabla$ MCF-7-T3411 $\ominus$ MCF-7
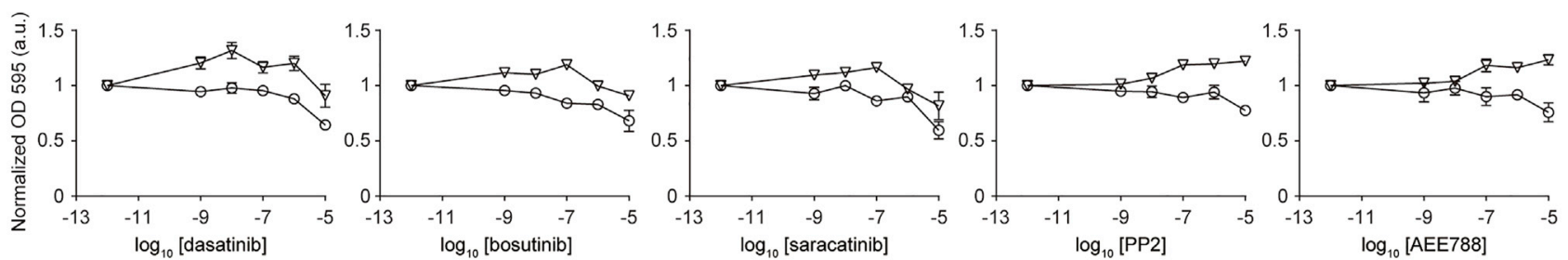

Figure 5. Kinase inhibitors activate a drug-resistant c-Src mutant

(A) Western blot to detect phospho-Y419 Src, unphosphorylated Y530 Src, Src (a rabbit polyclonal antibody was used), pY925 FAK, and FAK in an in vitro kinase assay. Inhibitors enhanced the autophosphorylation of c-Src-T338I and phosphorylation of FAK without dephosphorylation of pY527 (left panel). With wild-type c-Src, the phosphorylation of C-Src and FAK was suppressed by inhibitors (right panel). Src ${ }^{\text {ACT }}$ indicates C-Src-K249E-P250E-Y527F. Dasatinib, saracatinib, and bosutinib were used at $100 \mathrm{nM}$. PP2 and NVP-AEE788 (AEE788) were used at $10 \mu \mathrm{M}$. DMSO was used at $0.1 \%$. 
of c-Src-T338I in complex with FAK. In addition, our results indicate that this inhibitor-induced relief of c-Src autoinhibition may not require the dephosphorylation of $Y 527$, which is widely believed to be the mechanism for the autoinhibition release.

Treatment with Src inhibitors and the RTK inhibitor remarkably enhanced the localization of Grb2-EGFP to FAs in cells co-expressing the drug-resistant c-Src-T338I mutant, whereas the treatment decreased the localization of Grb2-EGFP in cells expressing wild-type c-Src (Figures 5C and S4A; Video S5). Next, we tested whether inhibitors activate drug-resistant c-Src expressed at the endogenous level, leading to the activation of Src-FAK-Erk signaling. To this end, we established MCF-7 cell lines harboring the T341I mutation in the endogenous SRC gene using CRISPR-Cas9 (Figure 5D). Treatment with inhibitors induced the activation of c-Src, FAK, and Erk in the cells harboring the c-Src T341I mutation, whereas the treatments did not induce the activation in parental MCF-7 cells (Figures 5E and S4B). It has been proposed that the dephosphorylation of $Y 530$ is a prerequisite for the activation of c-Src by phosphorylation at Y419 (Blencke et al., 2004; Xu et al., 1999). However, in our experiments, most of the inhibitors enhanced phosphorylation at Y419 in c-SrcT341I before the dephosphorylation of Y530 (Figures 5E and S4B). We tested whether inhibitor-induced paradoxical activation of Src may confer drug-resistant cell growth. High-affinity Src inhibitors dasatinib, bosutinib, and saracatinib at low doses promoted the proliferation of MCF-7-T341I cells compared with DMSO-treated MCF-7-T341I cells, although these inhibitors at high concentrations suppressed cell growth (Figure 5F). In contrast, PP2 and AEE788 enhanced the proliferation of MCF-7-T341I cells at high doses but not at low doses (Figure 5F). Importantly, the 4 Src inhibitors and the RTK inhibitor AEE788 did not promote the proliferation of parental MCF-7 cells expressing wild-type c-Src at any dose (Figure 5F). These results indicate that kinase inhibitors may paradoxically activate cell proliferation signaling in cells harboring drug-resistant mutations in the SRC gene.

\section{DISCUSSION}

We discovered that ATP-competitive Src kinase and RTK inhibitors turn into facilitators of drug-resistant c-Src mutants, which enhance Erk activity and promote cell proliferation. Even without a mutation, the binding of kinase inhibitors to wild-type c-Src induces its conformational change to resemble the active structure, leading to its association with FAK (Figure $6 \mathrm{~A}$ ). The resulting inhibitor/c-Src/FAK complex is not active, but when inhibitors do not tightly bind to c-Src, decreasing the inhibitor concentration may induce dissociation of the inhibitors from the complex while retaining $\mathrm{c}-\mathrm{Src}$ in an active open configuration, which allows
c-Src to readily phosphorylate FAK, leading to the activation of the FAK-Grb2-mediated Erk pathway (Figure 6B). We propose to call this unexpected activation of wild-type and mutant c-Src by Src and RTK inhibitors "paradoxical activation of Src."

Suppression of multiple kinases has been supposed to be effective in securely inhibiting oncogenic signals. The development of multi-drug therapies and multi-kinase-targeted drugs has been tried extensively (Wilhelm et al., 2006). However, our study revealed that kinase-targeted drugs may cause the unexpected activation of c-Src. This may be one reason why multi-kinase inhibitors have not been effective in cancer treatment. It has been thought that cancer cells that express drug-resistant kinases do not respond to drugs and that drugs have no effect on such cells. It has also been believed that the paradoxical mechanism by which drugs activate cancer growth signals is via the activation of molecules that are not supposed to be activated (Packer et al., 2011). However, our study shows that drugs directly activate drug-resistant target kinases. Drug-resistant mutations thus act as growth signal promoters, and not just drug-insensitive variants (Figure 6C). This indicates that drugs that aim to suppress cancer development, if cells have a drug-resistant mutation, may propagate a signal that exacerbates the cancer growth. Our research raises further alarms. These anti-cancer drugs, typically ATPcompetitive kinase inhibitors, are also able to activate normal cSrc. Intramolecular attachment of the C-terminal pY527 maintains a closed inactive conformation of c-Src and rarely results in an open conformation, which is referred to as a "self-suppressing function." Our study revealed that ATP-competitive kinase inhibitors allow c-Src to remarkably shift to the open conformation state, while pY527 is maintained (Figure 6A). Such an open conformational change induced by ATP-competitive kinase inhibitors appears to be similarly induced in c-Abl (Fujita et al., 2009; Skora et al., 2013; Sonti et al., 2018), Akt1 (Okuzumi et al., 2009), and Raf (Karoulia et al., 2016; Holderfield et al., 2013; Jin et al., 2017), suggesting that the conformational change may be common to kinases with self-suppressing functions. The open conformation may allow such kinases to bind their cognate targets and activate them when the drug concentration decreases. These results raise the possibility that kinase inhibitors, which aim to treat cancer, can evoke a growth signal that makes the cancer worse. To develop really effective anti-cancer drugs, we should understand the molecular mechanisms underlying the conformational changes of such kinases and overcome their paradoxical activation.

\section{STAR $\star$ METHODS}

Detailed methods are provided in the online version of this paper and include the following:

(B) In vitro binding assay of recombinant $6 \times$ His-tagged c-Src-T338I and GST-tagged phosphorylated Y397 FAK ${ }^{362-411}$ treated with inhibitors. The binding assay was monitored by the AlphaScreen assay. Data are presented as the means \pm SEMs $(n=3)$.

(C) Dasatinib enhanced localization of Grb2-EGFP at FAs in XTC cells expressing c-Src-T338I-TagRFP-T, but not in cells expressing c-Src-TagRFP-T. Scale bars, $5 \mu \mathrm{m}$. See also Figure S5 and Video S5.

(D) Chromatograph from Sanger sequencing of the SRC locus in MCF-7 and MCF-7-Tc-Src-T341I cells.

(E) Western blot analysis of phospho-Y419 Src, unphosphorylated Y530 Src, Src (a mouse monoclonal antibody was used), pY925-FAK, FAK, phospho-Erk (pErk), and Erk1\&2 (Erk) in MCF-7-c-Src-T341I and MCF-7 cell lines treated with dasatinib or 0.1\% DMSO (indicated by “-") for 30 min. See also Figure S5. (F) Src inhibitors and an RTK inhibitor enhanced proliferation of MCF-7 cells expressing drug-resistant c-Src-T341I. Cell proliferation was measured by optical density (OD) $595 \mathrm{~nm}$ of crystal violet staining. Cell growth was determined in triplicate. Data are presented as the means \pm SEMs $(\mathrm{n}=3$ ). 
A

c-Src

in closed state

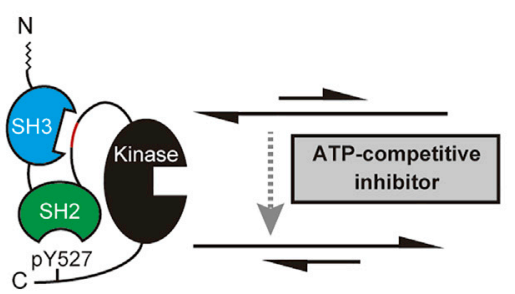

Inhibitor-induced c-Src Inhibitor/c-Src/FAK in open state

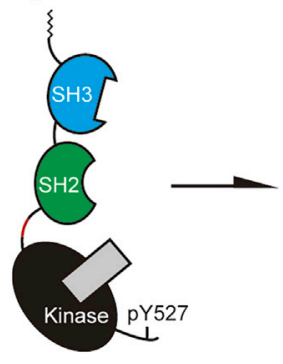

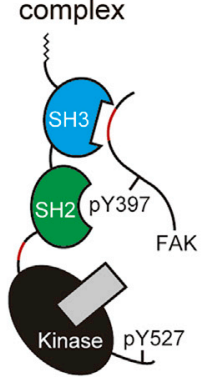

B

Inhibitor/c-Src/FAK complex

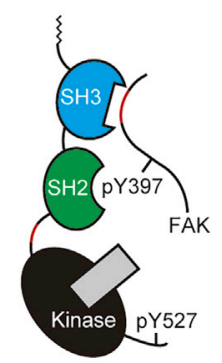

C

Inhibitor/drug resistant c-Src mutant/FAK

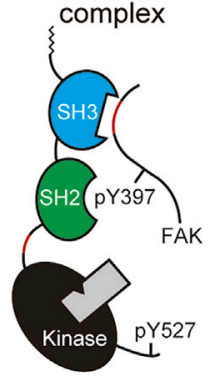

Fast dissocaiton of inhibitor promotes drug resistant c-Src mutant/FAK complex

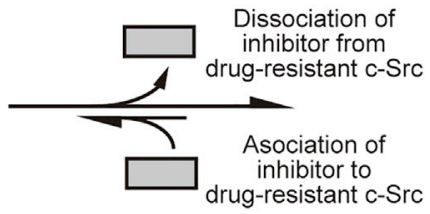

Washout promotes c-Src/FAK complex formation
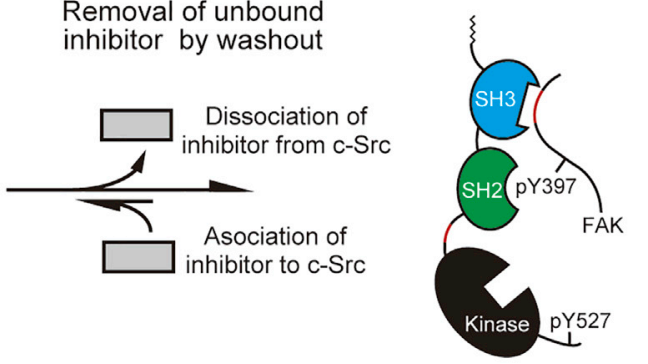

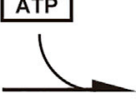

Treatment and washout of inhibitor induce paradoxical phosphorylation of C-Src and FAK

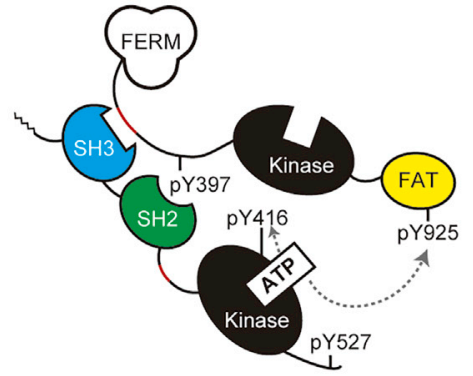

Drug resistant c-Src mutant IFAKcomplex
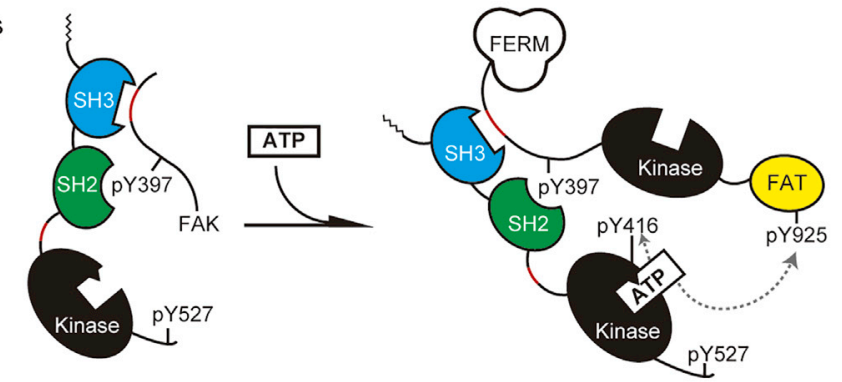

Figure 6. Model for inhibitor-induced paradoxical activation of c-Src

(A) Inhibitor-induced relief of autoinhibition of c-Src, leading to binding of c-Src to FAK. Relief of autoinhibited c-Src is triggered by the dephosphorylation of Tyr527 at the $\mathrm{C}$ terminus under normal conditions. However, ATP-competitive inhibitors shift the equilibrium and may disrupt the internal interaction of both the SH3 and SH2 domains in c-Src, even when phosphorylation at Tyr-527 remains intact.

(B) Treatment and washout of low-affinity inhibitors of c-Src induce paradoxical activation of c-Src. After the inhibitor/c-Src/FAK complex is formed, the removal of inhibitors by washout may dissociate inhibitors from the c-Src/FAK complex, and consequently c-Src phosphorylates c-Src and FAK. In contrast, high-affinity inhibitors may remain bound to c-Src after inhibitor washout, which may prevent paradoxical activation.

(C) Src and RTK inhibitors activate drug-resistant mutant c-Src. The reduced affinity of drug-resistant c-Src mutants for inhibitors accelerates dissociation of inhibitors from the inhibitor-induced c-Src/FAK complex. Even in the continuous presence of high-affinity inhibitors, drug-resistant c-Src is paradoxically activated to initiate the Grb2-Erk signaling pathway by phosphorylating Tyr-925 in FAK.

- KEY RESOURCES TABLE

- RESOURCE AVAILABILITY

○ Lead contact

Materials availability

Data and code availability

- EXPERIMENTAL MODEL AND SUBJECT DETAILS

Cell lines and generation of MCF-7-T341I cells
- METHOD DETAILS

Plasmids and reagents

$\circ$ Live-cell imaging

Imaging analysis

O Protein expression and purification

- AlphaScreen in vitro binding assay

o In vitro kinase assay 


\section{Western blotting}

○ Proliferation assay

- QUANTIFICATION AND STATISTICAL ANALYSIS

\section{SUPPLEMENTAL INFORMATION}

Supplemental information can be found online at https://doi.org/10.1016/j. celrep.2021.108876.

\section{ACKNOWLEDGMENTS}

We thank Dr. Akira Kakizuka for advising, Drs. Hiroto Katoh and Yasuyuki Fujita for MCF-7 cells, and Dr. Yunfeng Yuan for initial c-Abl-related experiments. This work was supported by CREST, JST (to N.W., grant no. JPMJCR15G5) and JSPS KAKENHI grant no. JP19H01020 (to N.W.).

\section{AUTHOR CONTRIBUTIONS}

N.W. and M.H. designed the experiments. M.H., K.I., M.M., and R.K. performed the expression of fluorescent proteins and live-cell imaging. M.H. performed the quantitative analysis of live-cell imaging, the protein purification, the in vitro binding assays, the in vitro kinase assays, the establishment of MCF-7-C-Src-T341/ knockin cell lines, the western blots, and the proliferation assays. A.T.-K. provided the reagents and suggestions. N.W. and M.H. wrote the manuscript.

\section{DECLARATION OF INTERESTS}

The authors declare no competing interests.

Received: October 15, 2020

Revised: December 29, 2020

Accepted: February 25, 2021

Published: March 23, 2021

\section{SUPPORTING CITATIONS}

The following references appear in the Supplemental information: Karaman et al. (2008).

\section{REFERENCES}

Apsel, B., Blair, J.A., Gonzalez, B., Nazif, T.M., Feldman, M.E., Aizenstein, B., Hoffman, R., Williams, R.L., Shokat, K.M., and Knight, Z.A. (2008). Targeted polypharmacology: discovery of dual inhibitors of tyrosine and phosphoinositide kinases. Nat. Chem. Biol. 4, 691-699.

Azam, M., Seeliger, M.A., Gray, N.S., Kuriyan, J., and Daley, G.Q. (2008). Activation of tyrosine kinases by mutation of the gatekeeper threonine. Nat. Struct. Mol. Biol. 15, 1109-1118.

Bagrodia, S., Taylor, S.J., and Shalloway, D. (1993). Myristylation is required for Tyr-527 dephosphorylation and activation of pp60c-src in mitosis. Mol. Cell. Biol. 13, 1464-1470.

Bain, J., Plater, L., Elliott, M., Shpiro, N., Hastie, C.J., McLauchlan, H., Klevernic, I., Arthur, J.S.C., Alessi, D.R., and Cohen, P. (2007). The selectivity of protein kinase inhibitors: a further update. Biochem. J. 408, 297-315.

Bear, J.E., Loureiro, J.J., Libova, I., Fässler, R., Wehland, J., and Gertler, F.B. (2000). Negative regulation of fibroblast motility by Ena/VASP proteins. Cell $101,717-728$

Bishop, A.C., Ubersax, J.A., Petsch, D.T., Matheos, D.P., Gray, N.S., Blethrow, J., Shimizu, E., Tsien, J.Z., Schultz, P.G., Rose, M.D., et al. (2000). A chemical switch for inhibitor-sensitive alleles of any protein kinase. Nature 407 , 395-401.

Blencke, S., Zech, B., Engkvist, O., Greff, Z., Orfi, L., Horváth, Z., Kéri, G., UIIrich, A., and Daub, H. (2004). Characterization of a conserved structural deter- minant controlling protein kinase sensitivity to selective inhibitors. Chem Biol. 11, 691-701.

Boschelli, D.H., Ye, F., Wang, Y.D., Dutia, M., Johnson, S.L., Wu, B., Miller, K., Powell, D.W., Yaczko, D., Young, M., et al. (2001). Optimization of 4-phenylamino-3-quinolinecarbonitriles as potent inhibitors of Src kinase activity. J. Med. Chem. 44, 3965-3977.

Brunton, V.G., Avizienyte, E., Fincham, V.J., Serrels, B., Metcalf, C.A., 3rd, Sawyer, T.K., and Frame, M.C. (2005). Identification of Src-specific phosphorylation site on focal adhesion kinase: dissection of the role of $\mathrm{Src} \mathrm{SH} 2$ and catalytic functions and their consequences for tumor cell behavior. Cancer Res. $65,1335-1342$

Cooper, J., and Giancotti, F.G. (2019). Integrin Signaling in Cancer: Mechanotransduction, Stemness, Epithelial Plasticity, and Therapeutic Resistance. Cancer Cell 35, 347-367.

Dai, Z., and Pendergast, A.M. (1995). Abi-2, a novel SH3-containing protein interacts with the c-Abl tyrosine kinase and modulates c-Abl transforming activity. Genes Dev. 9, 2569-2582.

Druker, B.J., Tamura, S., Buchdunger, E., Ohno, S., Segal, G.M., Fanning, S., Zimmermann, J., and Lydon, N.B. (1996). Effects of a selective inhibitor of the Abl tyrosine kinase on the growth of Bcr-Abl positive cells. Nat. Med. 2 , $561-566$.

Espada, J., and Martín-Pérez, J. (2017). An Update on Src Family of Nonreceptor Tyrosine Kinases Biology. Int. Rev. Cell Mol. Biol. 331, 83-122.

Fincham, V.J., Brunton, V.G., and Frame, M.C. (2000). The SH3 domain directs acto-myosin-dependent targeting of $\mathrm{v}$-Src to focal adhesions via phosphatidylinositol 3-kinase. Mol. Cell. Biol. 20, 6518-6536.

Fujita, A., Shishido, T., Yuan, Y., Inamoto, E., Narumiya, S., and Watanabe, N. (2009). Imatinib mesylate (STI571)-induced cell edge translocation of kinaseactive and kinase-defective Abelson kinase: requirements of myristoylation and src homology 3 domain. Mol. Pharmacol. 75, 75-84.

Girotti, M.R., Pedersen, M., Sanchez-Laorden, B., Viros, A., Turajlic, S., Niculescu-Duvaz, D., Zambon, A., Sinclair, J., Hayes, A., Gore, M., et al. (2013) Inhibiting EGF receptor or SRC family kinase signaling overcomes BRAF inhibitor resistance in melanoma. Cancer Discov. 3, 158-167.

Girotti, M.R., Lopes, F., Preece, N., Niculescu-Duvaz, D., Zambon, A., Davies, L., Whittaker, S., Saturno, G., Viros, A., Pedersen, M., et al. (2015). Paradoxbreaking RAF inhibitors that also target SRC are effective in drug-resistant BRAF mutant melanoma. Cancer Cell 27, 85-96.

Gonfloni, S., Frischknecht, F., Way, M., and Superti-Furga, G. (1999). Leucine 255 of Src couples intramolecular interactions to inhibition of catalysis. Nat. Struct. Biol. 6, 760-764.

Gorre, M.E., Mohammed, M., Ellwood, K., Hsu, N., Paquette, R., Rao, P.N., and Sawyers, C.L. (2001). Clinical resistance to STI-571 cancer therapy caused by BCR-ABL gene mutation or amplification. Science 293, 876-880.

Gottlieb-Abraham, E., Shvartsman, D.E., Donaldson, J.C., Ehrlich, M., Gutman, O., Martin, G.S., and Henis, Y.I. (2013). Src-mediated caveolin-1 phosphorylation affects the targeting of active Src to specific membrane sites. Mol. Biol. Cell 24, 3881-3895.

Green, T.P., Fennell, M., Whittaker, R., Curwen, J., Jacobs, V., Allen, J., Logie, A., Hargreaves, J., Hickinson, D.M., Wilkinson, R.W., et al. (2009). Preclinical anticancer activity of the potent, oral Src inhibitor AZD0530. Mol. Oncol. 3 , 248-261.

Hamidi, H., and Ivaska, J. (2018). Every step of the way: integrins in cancer progression and metastasis. Nat. Rev. Cancer 18, 533-548.

Hatzivassiliou, G., Song, K., Yen, I., Brandhuber, B.J., Anderson, D.J., Alvarado, R., Ludlam, M.J.C., Stokoe, D., Gloor, S.L., Vigers, G., et al. (2010). RAF inhibitors prime wild-type RAF to activate the MAPK pathway and enhance growth. Nature 464, 431-435.

Heim, J.B., Squirewell, E.J., Neu, A., Zocher, G., Sominidi-Damodaran, S. Wyles, S.P., Nikolova, E., Behrendt, N., Saunte, D.M., Lock-Andersen, J., et al. (2017). Myosin-1E interacts with FAK proline-rich region 1 to induce fibronectin-type matrix. Proc. Natl. Acad. Sci. USA 114, 3933-3938. 
Cell Reports Article $\infty$ CellPress

OPEN ACCESS
Hildebrand, J.D., Schaller, M.D., and Parsons, J.T. (1993). Identification of sequences required for the efficient localization of the focal adhesion kinase, pp125FAK, to cellular focal adhesions. J. Cell Biol. 123, 993-1005.

Hirata, E., Girotti, M.R., Viros, A., Hooper, S., Spencer-Dene, B., Matsuda, M., Larkin, J., Marais, R., and Sahai, E. (2015). Intravital imaging reveals how BRAF inhibition generates drug-tolerant microenvironments with high integrin $\beta 1 /$ FAK signaling. Cancer Cell 27, 574-588.

Holderfield, M., Merritt, H., Chan, J., Wallroth, M., Tandeske, L., Zhai, H., Tellew, J., Hardy, S., Hekmat-Nejad, M., Stuart, D.D., et al. (2013). RAF inhibitors activate the MAPK pathway by relieving inhibitory autophosphorylation. Cancer Cell 23, 594-602.

Horzum, U., Ozdil, B., and Pesen-Okvur, D. (2014). Step-by-step quantitative analysis of focal adhesions. MethodsX. 1, 56-59.

Jin, T., Lavoie, H., Sahmi, M., David, M., Hilt, C., Hammell, A., and Therrien, M. (2017). RAF inhibitors promote RAS-RAF interaction by allosterically disrupting RAF autoinhibition. Nat. Commun. 8, 1211.

Johannessen, C.M., Boehm, J.S., Kim, S.Y., Thomas, S.R., Wardwell, L., Johnson, L.A., Emery, C.M., Stransky, N., Cogdill, A.P., Barretina, J., et al. (2010). COT drives resistance to RAF inhibition through MAP kinase pathway reactivation. Nature 468, 968-972.

Joseph, E.W., Pratilas, C.A., Poulikakos, P.I., Tadi, M., Wang, W., Taylor, B.S., Halilovic, E., Persaud, Y., Xing, F., Viale, A., et al. (2010). The RAF inhibitor PLX4032 inhibits ERK signaling and tumor cell proliferation in a V600E BRAF-selective manner. Proc. Natl. Acad. Sci. USA 107, 14903-14908.

Karaman, M.W., Herrgard, S., Treiber, D.K., Gallant, P., Atteridge, C.E., Campbell, B.T., Chan, K.W., Ciceri, P., Davis, M.I., Edeen, P.T., et al. (2008). A quantitative analysis of kinase inhibitor selectivity. Nat. Biotechnol. 26, 127-132.

Karoulia, Z., Wu, Y., Ahmed, T.A., Xin, Q., Bollard, J., Krepler, C., Wu, X., Zhang, C., Bollag, G., Herlyn, M., et al. (2016). An Integrated Model of RAF Inhibitor Action Predicts Inhibitor Activity against Oncogenic BRAF Signaling. Cancer Cell 30, 485-498.

Kim, L.C., Song, L., and Haura, E.B. (2009). Src kinases as therapeutic targets for cancer. Nat. Rev. Clin. Oncol. 6, 587-595.

Koshman, Y.E., Engman, S.J., Kim, T., lyengar, R., Henderson, K.K., and Samarel, A.M. (2010). Role of FRNK tyrosine phosphorylation in vascular smooth muscle spreading and migration. Cardiovasc. Res. 85, 571-581.

Krishnamurty, R., Brigham, J.L., Leonard, S.E., Ranjitkar, P., Larson, E.T., Dale, E.J., Merritt, E.A., and Maly, D.J. (2013). Active site profiling reveals coupling between domains in SRC-family kinases. Nat. Chem. Biol. 9, 43-50.

Levinson, N.M., and Boxer, S.G. (2014). A conserved water-mediated hydrogen bond network defines bosutinib's kinase selectivity. Nat. Chem. Biol. 10, 127-132.

Lietha, D., Cai, X., Ceccarelli, D.F.J., Li, Y., Schaller, M.D., and Eck, M.J. (2007). Structural basis for the autoinhibition of focal adhesion kinase. Cell $129,1177-1187$.

Liu, X., Marengere, L.E., Koch, C.A., and Pawson, T. (1993). The v-Src SH3 domain binds phosphatidylinositol 3'-kinase. Mol. Cell. Biol. 13, 5225-5232.

Maruoka, M., Sato, M., Yuan, Y., Ichiba, M., Fujii, R., Ogawa, T., Ishida-Kitagawa, N., Takeya, T., and Watanabe, N. (2012). Abl-1-bridged tyrosine phosphorylation of VASP by Abelson kinase impairs association of VASP to focal adhesions and regulates leukaemic cell adhesion. Biochem. J. 441, 889-899. Millius, A., Watanabe, N., and Weiner, O.D. (2012). Diffusion, capture and recycling of SCAR/WAVE and Arp2/3 complexes observed in cells by singlemolecule imaging. J. Cell Sci. 125, 1165-1176.

Nakagawa, H., Miki, H., Nozumi, M., Takenawa, T., Miyamoto, S., Wehland, J., and Small, J.V. (2003). IRSp53 is colocalised with WAVE2 at the tips of protruding lamellipodia and filopodia independently of Mena. J. Cell Sci. 116, 25772583.

O'Hare, T., Walters, D.K., Stoffregen, E.P., Jia, T., Manley, P.W., Mestan, J., Cowan-Jacob, S.W., Lee, F.Y., Heinrich, M.C., Deininger, M.W.N., and Druker, B.J. (2005). In vitro activity of Bcr-Abl inhibitors AMN107 and BMS-354825 against clinically relevant imatinib-resistant Abl kinase domain mutants. Cancer Res. 65, 4500-4505.
Oikawa, T., Itoh, T., and Takenawa, T. (2008). Sequential signals toward podosome formation in NIH-src cells. J. Cell Biol. 182, 157-169.

Okamura, H., and Resh, M.D. (1995). p80/85 cortactin associates with the Src SH2 domain and colocalizes with v-Src in transformed cells. J. Biol. Chem. 270, 26613-26618.

Okuzumi, T., Fiedler, D., Zhang, C., Gray, D.C., Aizenstein, B., Hoffman, R., and Shokat, K.M. (2009). Inhibitor hijacking of Akt activation. Nat. Chem. Biol. 5, 484-493.

Packer, L.M., Rana, S., Hayward, R., O'Hare, T., Eide, C.A., Rebocho, A., Heidorn, S., Zabriskie, M.S., Niculescu-Duvaz, I., Druker, B.J., et al. (2011). Nilotinib and MEK inhibitors induce synthetic lethality through paradoxical activation of RAF in drug-resistant chronic myeloid leukemia. Cancer Cell 20, 715-727.

Papa, F.R., Zhang, C., Shokat, K., and Walter, P. (2003). Bypassing a kinase activity with an ATP-competitive drug. Science 302, 1533-1537.

Patwardhan, P., and Resh, M.D. (2010). Myristoylation and membrane binding regulate c-Src stability and kinase activity. Mol. Cell. Biol. 30, 4094-4107.

Poulikakos, P.I., Zhang, C., Bollag, G., Shokat, K.M., and Rosen, N. (2010). RAF inhibitors transactivate RAF dimers and ERK signalling in cells with wild-type BRAF. Nature 464, 427-430.

Qiao, Y., Molina, H., Pandey, A., Zhang, J., and Cole, P.A. (2006). Chemical rescue of a mutant enzyme in living cells. Science 311, 1293-1297.

Roskoski, R., Jr. (2015). Src protein-tyrosine kinase structure, mechanism, and small molecule inhibitors. Pharmacol. Res. 94, 9-25.

Schindler, T., Bornmann, W., Pellicena, P., Miller, W.T., Clarkson, B., and Kuriyan, J. (2000). Structural mechanism for STI-571 inhibition of abelson tyrosine kinase. Science 289, 1938-1942.

Schlaepfer, D.D., Hanks, S.K., Hunter, T., and Geer, P. Van Der. (1994). Integrin-mediated signal transduction linked to Ras pathway by GRB2 binding to focal adhesion kinase. Nature 372, 786-791.

Shi, Y., Alin, K., and Goff, S.P. (1995). Abl-interactor-1, a novel SH3 protein binding to the carboxy-terminal portion of the Abl protein, suppresses $\mathrm{v}$-abl transforming activity. Genes Dev. 9, 2583-2597.

Skora, L., Mestan, J., Fabbro, D., Jahnke, W., and Grzesiek, S. (2013). NMR reveals the allosteric opening and closing of Abelson tyrosine kinase by ATP-site and myristoyl pocket inhibitors. Proc. Natl. Acad. Sci. USA 110, E4437-E4445.

Sonti, R., Hertel-Hering, I., Lamontanara, A.J., Hantschel, O., and Grzesiek, S. (2018). ATP site ligands determine the assembly state of the Abelson kinase regulatory core via the activation loop conformation. J. Am. Chem. Soc. 140, 1863-1869.

Sulzmaier, F.J., Jean, C., and Schlaepfer, D.D. (2014). FAK in cancer: mechanistic findings and clinical applications. Nat. Rev. Cancer 14, 598-610.

Tanji, M., Ishizaki, T., Ebrahimi, S., Tsuboguchi, Y., Sukezane, T., Akagi, T., Frame, M.C., Hashimoto, N., Miyamoto, S., and Narumiya, S. (2010). mDia1 targets $\mathrm{v}$-Src to the cell periphery and facilitates cell transformation, tumorigenesis, and invasion. Mol. Cell. Biol. 30, 4604-4615.

Traxler, P., Allegrini, P.R., Brandt, R., Brueggen, J., Cozens, R., Fabbro, D., Grosios, K., Lane, H.A., McSheehy, P., Mestan, J., et al. (2004). AEE788: a dual family epidermal growth factor receptor/ErbB2 and vascular endothelial growth factor receptor tyrosine kinase inhibitor with antitumor and antiangiogenic activity. Cancer Res. 64, 4931-4941.

Van Etten, R.A. (1999). Cycling, stressed-out and nervous: Cellular functions of c-Abl. Trends Cell Biol. 9, 179-186.

Verderame, M.F. (1997). pp60v-src transformation of rat cells but not chicken cells strongly correlates with low-affinity phosphopeptide binding by the $\mathrm{SH} 2$ domain. Mol. Biol. Cell 8, 843-854.

Watanabe, N. (2012). Fluorescence single-molecule imaging of actin turnover and regulatory mechanisms. Methods Enzymol. 505, 219-232.

Watanabe, N., and Mitchison, T.J. (2002). Single-molecule speckle analysis of actin filament turnover in lamellipodia. Science 295, 1083-1086. 
Wilhelm, S., Carter, C., Lynch, M., Lowinger, T., Dumas, J., Smith, R.A., Schwartz, B., Simantov, R., and Kelley, S. (2006). Discovery and development of sorafenib: a multikinase inhibitor for treating cancer. Nat. Rev. Drug Discov. 5, 835-844.

Xu, W., Doshi, A., Lei, M., Eck, M.J., and Harrison, S.C. (1999). Crystal structures of c-Src reveal features of its autoinhibitory mechanism. Mol. Cell. 3, 629-638.

Xu, W., Harrison, S.C., and Eck, M.J. (1997). Three-dimensional structure of the tyrosine kinase c-Src. Nature 385, 595-602.

Yamashiro, S., and Watanabe, N. (2014). A new link between the retrograde actin flow and focal adhesions. J. Biochem. 156, 239-248.

Yeatman, T.J. (2004). A renaissance for SRC. Nat. Rev. Cancer 4, 470-480.

Yeo, M.G., Partridge, M.A., Ezratty, E.J., Shen, Q., Gundersen, G.G., and Marcantonio, E.E. (2006). Src SH2 arginine 175 is required for cell motility: specific focal adhesion kinase targeting and focal adhesion assembly function. Mol. Cell. Biol. 26, 4399-4409.

Zhang, S., and Yu, D. (2012). Targeting Src family kinases in anti-cancer therapies: turning promise into triumph. Trends Pharmacol. Sci. 33, 122-128.

Zhang, S., Huang, W.C., Li, P., Guo, H., Poh, S.B., Brady, S.W., Xiong, Y., Tseng, L.M., Li, S.H., Ding, Z., et al. (2011). Combating trastuzumab resistance by targeting SRC, a common node downstream of multiple resistance pathways. Nat. Med. 17, 461-469.

Zhang, C., Lopez, M.S., Dar, A.C., Ladow, E., Finkbeiner, S., Yun, C.H., Eck, M.J., and Shokat, K.M. (2013). Structure-guided inhibitor design expands the scope of analog-sensitive kinase technology. ACS Chem. Biol. 8, 19311938. 


\section{STAR $\star$ METHODS}

\section{KEY RESOURCES TABLE}

\begin{tabular}{|c|c|c|}
\hline REAGENT or RESOURCE & SOURCE & IDENTIFIER \\
\hline \multicolumn{3}{|l|}{ Antibodies } \\
\hline $\begin{array}{l}\text { Rabbit polyclonal anti-phospho-Tyr416 } \\
\text { (419) Src }\end{array}$ & Cell signaling technology & Cat\#2101; RRID: AB_331697 \\
\hline $\begin{array}{l}\text { Rabbit polyclonal anti-non-phospho- } \\
\text { Tyr527 (530) Src }\end{array}$ & Cell signaling technology & Cat\#2107; RRID: AB_331081 \\
\hline Mouse monoclonal anti-Src (clone L4A1) & Cell signaling technology & Cat\#2110; RRID: AB_10691385 \\
\hline Rabbit polyclonal anti-Src (clone 32G6) & Cell signaling technology & Cat\#2113; RRID: AB_2106051 \\
\hline Rabbit polyclonal anti-pY925 FAK & Abcam & Cat\#ab38512; RRID: AB_732302 \\
\hline Rabbit polyclonal anti-FAK & Cell signaling technology & Cat\#3285; RRID: AB_2269034 \\
\hline $\begin{array}{l}\text { Mouse monoclonal anti- phospho-Thr202/ } \\
\text { Tyr204-Erk1/2 (clone E10) }\end{array}$ & Cell signaling technology & Cat\#9106; RRID: AB_331768 \\
\hline Rabbit polyclonal anti-Erk1 & Sigma Aldrich & Cat\#M7927; RRID: AB_260665 \\
\hline Rabbit polyclonal anti-His-tag & MBL & Cat\#PM032; RRID: AB_10209426 \\
\hline Rabbit polyclonal anti-GFP & Abcam & Cat\#ab290 ; RRID: AB_303395 \\
\hline \multicolumn{3}{|l|}{ Bacterial and virus strains } \\
\hline BL21(DE3) & New England Biolabs & Cat\#C2527I \\
\hline $\mathrm{DH} 5 \alpha$ & TOYOBO Life Science & Cat\#DNA-913 \\
\hline \multicolumn{3}{|l|}{ Chemicals, peptides, and recombinant proteins } \\
\hline DYKDDDK peptide & Wako & Cat\#044-30951 \\
\hline Dasatinib & LC laboratories & Cat\#D-3307 \\
\hline Bosutinib & Sigma Aldrich & Cat\#PZ0192 \\
\hline Saracatinib & Selleck Chemicals & Cat\#S1006 \\
\hline PP2 & Selleck Chemicals & Cat\#S7008 \\
\hline NVP-AEE788 & Selleck Chemicals & Cat\#S1486 \\
\hline AMP-PNP & Sigma Aldrich & Cat\#10102547001 \\
\hline 1-NA-PP1 & Sigma Aldrich & Cat\#529579 \\
\hline 1-NM-PP1 & Sigma Aldrich & Cat\#529581 \\
\hline imatinib & Selleck Chemicals & Cat\#S2475 \\
\hline ATP & nacalai Tesque & Cat\#01072-24 \\
\hline GeneArt Platinum Cas9 nuclease & Thermo Fisher SCIENTIFIC & Cat\#B25640 \\
\hline GST-Prescission Protease & GE healthcare & Cat\#27084301 \\
\hline \multicolumn{3}{|l|}{ Experimental models: cell lines } \\
\hline XTC & Watanabe and Mitchison, 2002 & Watanabe and Mitchison, 2002 \\
\hline HEK293T & Maruoka et al., 2012 & Maruoka et al., 2012 \\
\hline MCF-7 & Drs. Hiroto Katoh and Yasuyuki Fujita & N/A \\
\hline MCF-7-T341I & This Study & $\mathrm{N} / \mathrm{A}$ \\
\hline \multicolumn{3}{|l|}{ Oligonucleotides } \\
\hline $\begin{array}{l}\text { See Table S2 for sgRNA and } \\
\text { oligonucleotides }\end{array}$ & $\begin{array}{l}\text { Sigma Aldrich or Thermo Fisher } \\
\text { SCIENTIFIC }\end{array}$ & N/A \\
\hline \multicolumn{3}{|l|}{ Recombinant DNA } \\
\hline pEGFP-N3 & CloneTech Laboratories & Cat\#6080-1 \\
\hline pEGFP-N1 & CloneTech Laboratories & Cat\#6085-1 \\
\hline pEGFP-C1 & CloneTech Laboratories & Cat\#6084-1 \\
\hline delCMV-EGFP-N3 & Watanabe and Mitchison, 2002 & Watanabe and Mitchison, 2002 \\
\hline pEGFP-N3-3 $\times$ Flag & This paper & N/A \\
\hline
\end{tabular}

(Continued on next page) 


\begin{tabular}{|c|c|c|}
\hline Continued & & \\
\hline REAGENT or RESOURCE & SOURCE & IDENTIFIER \\
\hline pFlag-6p-C1 & This paper & $\mathrm{N} / \mathrm{A}$ \\
\hline pFlag-6p-C1-6 $\times$ His & This paper & N/A \\
\hline pTagRFP-T-C1 & Evrogen & Cat\#FP141 \\
\hline pTagRFP-T-N3 & This paper & N/A \\
\hline pGEX4T-1 & GE helathcare & Cat\#28954549 \\
\hline pEGFP-N1-c-Src & Tanji et al., 2010 & Tanji et al., 2010 \\
\hline pEGFP-N1-c-Src-G2A & This Study & N/A \\
\hline pEGFP-N1-c-Src-W118A & This Study & N/A \\
\hline pEGFP-N1-c-Src-R175A & This Study & N/A \\
\hline pEGFP-N1-c-Src-K295M & This Study & N/A \\
\hline pEGFP-N1-c-Src-T338I & This Study & N/A \\
\hline pEGFP-N1-c-Src-T338G & This Study & N/A \\
\hline pEGFP-N1-c-Src-W118A-R175A & This Study & N/A \\
\hline pEGFP-N3-c-Src-K249E-P250E-Y527F & This Study & N/A \\
\hline pTagRFP-T-N3-c-Src & This Study & $\mathrm{N} / \mathrm{A}$ \\
\hline pTagRFP-T-N3-c-Src-T338I & This Study & N/A \\
\hline pTagRFP-T-N3-c-Src-R388A-Y527F & This Study & N/A \\
\hline pFlag-6p-C1-c-Src & This Study & N/A \\
\hline pFlag-6p-C1-c-Src-T338I & This Study & $\mathrm{N} / \mathrm{A}$ \\
\hline pFlag-6p-C1-c-Src-K249E-P250E-Y527F & This Study & N/A \\
\hline pFlag-6p-C1-6 $\times$ His-Src & This Study & $\mathrm{N} / \mathrm{A}$ \\
\hline pFlag-6p-C1-6 $\times$ His-Src-T338I & This Study & $\mathrm{N} / \mathrm{A}$ \\
\hline Human FAK cDNA & DNASU & Cat\#HsCD00021642 \\
\hline pEGFP-C1-FAK & This Study & N/A \\
\hline pTagRFP-T-C1-FAK & This Study & $\mathrm{N} / \mathrm{A}$ \\
\hline pTagRFP-T-C1-FAT & This Study & N/A \\
\hline pTagRFP-T-C1-FAK-362-686 & This Study & N/A \\
\hline pFlag-C1-FAK-412-686 & This Study & N/A \\
\hline pFlag-C1- $\Delta$ FERM & This Study & N/A \\
\hline pGEX4T-1-FAK-362-411 & This Study & $\mathrm{N} / \mathrm{A}$ \\
\hline pGEX4T-1-FAK-362-411-P371A-P374A & This Study & $\mathrm{N} / \mathrm{A}$ \\
\hline pJ3H PTP-1B & Addgene & Cat\#8601 \\
\hline pGEX4T-1-PTP-1B-1-321 & This Study & $\mathrm{N} / \mathrm{A}$ \\
\hline Human Grb2 cDNA & Kazusa DNA & Cat\#FXC01485 \\
\hline delCMV-EGFP-N3-Grb2 & This Study & $\mathrm{N} / \mathrm{A}$ \\
\hline delCMV-mPlum-C1-Vinculin & Yamashiro and Watanabe, 2014 & Yamashiro and Watanabe, 2014 \\
\hline Human c-Abl cDNA & MyBioSource & Cat\#MBS1267867 \\
\hline pEGFP-N3-3 $\times$ Flag-Human c-Abl-1-632 & This paper & $\mathrm{N} / \mathrm{A}$ \\
\hline pFlag-CMV-6c-Human Abi-1 & Maruoka et al., 2012 & Maruoka et al., 2012 \\
\hline pGEX4T-1-Human Abi-1 & This paper & N/A \\
\hline Xenopus Abi-1 & IMAGE Consortium & Cat\#6958745 \\
\hline pmRFP1-C1- Xenopus Abi1 & This paper & N/A \\
\hline pmRFP1-C1- Xenopus Abi1-mito & This paper & $\mathrm{N} / \mathrm{A}$ \\
\hline Xenopus WAVE2 & IMAGE Consortium & Cat\#6870338 \\
\hline pFlag-Xenopus WAVE2-mito & This paper & $\mathrm{N} / \mathrm{A}$ \\
\hline \multicolumn{3}{|l|}{ Software and algorithms } \\
\hline ImgaeJ & $\mathrm{NIH}$ & https://imagej.nih.gov/ij/ \\
\hline GraphPadPrism 6 & GraphPad Software Inc. & https://www.graphpad.com/ \\
\hline Metamorph & MolecularDevices & https://www.moleculardevices.com/ \\
\hline
\end{tabular}




\begin{tabular}{|c|c|c|}
\hline \multicolumn{3}{|l|}{ Continued } \\
\hline REAGENT or RESOURCE & SOURCE & IDENTIFIER \\
\hline ACD/ChemSketch & $\begin{array}{l}\text { Advanced Chemistry Development, Inc. } \\
\text { (ACD/Labs) }\end{array}$ & $\begin{array}{l}\text { https://www.acdlabs.com/resources/ } \\
\text { freeware/chemsketch/ }\end{array}$ \\
\hline \multicolumn{3}{|l|}{ Other } \\
\hline Sodium Orthovanadate & Santa Cruz Biotechnology & Cat\#sc-3540 \\
\hline Ovalbumin & Sigma Aldrich & Cat\#A5503 \\
\hline BSA & Sigma Aldrich & Cat\#A7030 \\
\hline Sodium Fluoride & Wako & Cat\#192-01972 \\
\hline Bovine fibronectin & Sigma Aldrich & Cat\#F1141 \\
\hline PEI & Polysciences & Cat\#24765-1 \\
\hline Anti-DYKDDDDK tag antibody beads & Wako & Cat\#018-22783 \\
\hline GST-Accept beads & nacalai Tesque & Cat\#09277-72 \\
\hline Protease Inhibitor Cocktail (EDTA free) & nacalai Tesque & Cat\#03969-34 \\
\hline Leibovitz's L-15 (for cell culture) & Thermo Fisher Scientific & Cat\#11415-064 \\
\hline $\begin{array}{l}\text { Leibovitz's L-15 (for microscopy } \\
\text { observation) }\end{array}$ & nacalai Tesque & Custom Synthesis \\
\hline fetal calf serum & Thermo Fisher Scientific & Cat\#10270-106 \\
\hline DMEM & nacalai Tesque & Cat\#08459-35 \\
\hline Spin column & Thermo Fisher Scientific & Cat\#26147 \\
\hline AlphaScreen Protein A acceptor beads & PerkinElmer & Cat\#6760137 \\
\hline AlphaScreen glutathione-donor beads & PerkinElmer & Cat\#6765301 \\
\hline 384-well optiplate for AlphaScreen & PerkinElmer & Cat\#6007290 \\
\hline 2.5g/l-Trypsin/1mmol/l-EDTA Solution & nacalai Tesque & Cat\#35554-64 \\
\hline Envision 2105 & PerkinElmer & Cat\#2105 \\
\hline IX83 Inverted microscope & Olympus & IX83 Inverted microscope \\
\hline Evolve 512 & Photometrics & Evolve 512 \\
\hline Neon ${ }^{\mathrm{TM}}$ Transfection System & Thermo Fisher SCIENTIFIC & Cat\#MPK5000 \\
\hline Neon ${ }^{\mathrm{TM}}$ Transfection System $10 \mu \mathrm{l} \mathrm{Kit}$ & Thermo Fisher SCIENTIFIC & Cat\#MPK1096 \\
\hline
\end{tabular}

\section{RESOURCE AVAILABILITY}

\section{Lead contact}

Further information and requests for resources and reagents should be directed to and will be fulfilled by the lead contact, Naoki Watanabe (watanabe.naoki.4v@kyoto-u.ac.jp).

Materials availability

Plasmids and cell lines generated in this study are available from the lead contact with a completed Materials Transfer Agreement.

Data and code availability

This study did not generate any unique datasets or codes.

\section{EXPERIMENTAL MODEL AND SUBJECT DETAILS}

Cell lines and generation of MCF-7-T341I cells

Xenopus laevis XTC cells were maintained in 70\% Leibovitz's L15 medium (Thermo Fisher Scientific, 11415-064) containing 10\% fetal calf serum (FCS, Thermo Fisher Scientific, 10270-106) at 23 $\mathrm{C}$ in incubator (Fujita et al., 2009; Watanabe, 2012; Yamashiro and Watanabe, 2014). HEK293F, MCF-7 cells and MCF-7-T341I cells were maintained in DMEM (nacalai tesque, 08459-35) containing 10\% FCS at $37^{\circ} \mathrm{C}$ in humidified $95 \% \mathrm{CO}_{2}$ incubator. Plasmid transfection was carried out with polyethyleneimine (PEI) (Polysciences, 23966-1) (Fujita et al., 2009; Watanabe, 2012; Yamashiro and Watanabe, 2014). MCF-7-T341I cells were generated using CRISPR technology. Transfection of sgRNA, donor oligonucleotide and Cas9 recombinant protein was performed by electroporation with a Neon Transfection System (Invitrogen) as follows. MCF-7 cells $\left(1.2 \times 10^{5}\right.$ cells, $1150 \mathrm{~V}, 30 \mathrm{~ms}, 2$ pulses), $1 \mu \mathrm{g}$ GeneArt Platinum Cas 9 nuclease (ThermoFisher scientific, B25640), $1 \mu \mathrm{g}$ custom IVTgRNA (ThermoFisher scientific) and 100 pmol custom single-strand donor oligonucleotide (ThermoFisher scientific) were used for a $10 \mu \mathrm{L}$ reaction. Sequences of IVTgRNA and single-strand donor oligonucleotide were 
5'-GGAGCCCATTTACATCGTCA-3' and 5'-AGGTCATGAAGAAGCTGAGGCATGAGAAGCTGGTGCAGTTGTATGCTGTGGTTTCA GAGGAGCCCATTTACATCGTCATAGAGTACATGAGCAAGGGTGAGTCCTGGGCGGCCGGGGCA-3', respectively. Genome editing was allowed to proceed for 3 days, and limiting dilution was performed to isolate monoclonal cell lines. To confirm which clones harbored endogenous C-Src-T3411, genomic DNA was purified, and the target locus was amplified by PCR for sequencing. Forward and reverse primers for amplifying the c-Src locus were 5'-CACAGTCCCGACAACAGCTACTGG-3' and 5'-GAGAATTTGGCTCTACTGCATTC CACC- $3^{\prime}$, respectively.

\section{METHOD DETAILS}

\section{Plasmids and reagents}

pTagRFP-T-N3 was constructed by replacing the EGFP cassette with a TagRFP-T cassette (Evrogen) in pEGFP-N3 (ClonTech Laboratories). Chicken c-Src cDNA (Tanji et al., 2010) was amplified by PCR and subcloned into pEGFP-N1 (ClonTech Laboratories) or pTagRFP-T-N3. pFlag-C1 was constructed by replacing the EGFP cassette with a Flag sequence in pEGFP-C1. pFlag-6p-C1 was constructed by inserting a PreScission protease cleavage sequence (GE Healthcare) between the Flag sequence and the MCS in pFlag-C1. pFlag-6p-C1-6 $\times$ His was constructed by inserting a $6 \times$ His sequence between the PreScission protease cleavage sequence and the MCS in pFlag-6p-C1. Human FAK cDNA (GenBank accession number: BC035404) was purchased from DNASU and was amplified by PCR and subcloned into pEGFP-C1 (ClonTech Laboratories) or pTagRFP-T-C1. Deletion mutants encoding the FAK FAT domain (amino acids 861-1006), the c-Src binding site of FAK (amino acids 362-411), the FAK kinase domain (amino acids 412-686), $\triangle$ FERM (amino acids 362-1006) and the FERM and FAT deletion FAK (362-686) were amplified by PCR and subcloned into pTagRFP-T-C1, pGEX4T-1 or pFlag-C1, respectively. Point mutations in c-Src and FAK were generated by site-directed mutagenesis using KOD Plus (TOYOBO) and Dpnl (NEB). Human Grb2 cDNA (GeneBank accession number: BC000631) was obtained from Kazusa DNA Research Institute and was subcloned into delCMV-EGFP-N3 (Watanabe and Mitchison, 2002). Human vinculin cDNA was amplified by PCR and subcloned into delCMV-mPlum-C1 (Yamashiro and Watanabe, 2014). The human PTP-1B catalytic domain (amino acids 1-321) was amplified by PCR from pJ3H PTP-1B and subcloned into pGEX4T-1 (pJ3H PTP-1B was a gift from Ben Neel (Addgene plasmid \#8601; https://www.addgene.org/8601/.org; RRID:Addgene_8601)). The expression vectors for murine type IV c-Abl were described previously (Fujita et al., 2009). cDNA clones encoding Xenopus Abi-1 (GeneBank accession number: BC081178) and WAVE2 (GeneBank accession number: BC089121) were obtained from the IMAGE Consortium. Human type b c-Abl cDNA (GeneBank accession number: BC117451) was purchased from Open Biosystems. Human Abi-1 cDNA was described previously (Maruoka et al., 2012). pmRFP1-C1 was constructed by replacing the EGFP cassette in pEGFP-C1 with mRFP1 cDNA. Xenopus Abi-1 and WAVE2 were amplified by PCR and subcloned into pmRFP1-C1 and pFlag-C1, respectively. To generate mitochondria-anchoring constructs including mRFP1-Xenopus Abi-1-mito and Flag-WAVE2-mito, the fragment corresponding to the coding region of the mitochondrial-anchoring signal of Listeria ActA (amino acids 614-639) (Bear et al., 2000) was fused to the respective cDNAs in the expression vectors. pEGFP-N3-3 $\times$ Flag was constructed by replacing the EGFP cassette with an EGFP-3 $\times$ Flag cassette. Human c-Abl-1-623, encoding the $\mathrm{SH} 3, \mathrm{SH} 2$ and kinase domains, was amplified by PCR and subcloned into pEGFP-N3-3 $\times$ Flag. Human Abi-1 was amplified by PCR and subcloned into pGEX-4T-1. Dasatinib was purchased from LC laboratories. Saracatinib, bosutinib, NVP-AEE788 and PP2 were purchased from Selleck Chemicals. 1-NA-PP1 and 1-NM-PP1 were purchased from Merck Millipore. AMP-PNP was purchased from Sigma Aldrich. Chemical structures of compounds were written by ACD/ChemSketch.

\section{Live-cell imaging}

Live-cell imaging of XTC cells was carried out as described previously (Watanabe and Mitchison, 2002). Before observation of c-Src, FAK or Grb2, XTC cells were trypsinized and seeded onto a glass coverslip dually coated with poly-L-lysine $(10 \mu \mathrm{g} / \mathrm{ml})$ and bovine fibronectin ( $3 \mu \mathrm{g} / \mathrm{ml}$ Sigma-Aldrich, F1141) in 70\% riboflavin-free and phenol red-free custom Leibovitz's L15 medium (nacalai tesque, custom order) without FCS for 1 hour. For observation of cells expressing c-Abl, Abi-1 or WAVE2, a glass coverslip was coated with poly-L-lysine (1 mg/ml). The coverslip was mounted in a cell chamber (Thermo Fisher Scientific, A7816). Images were acquired using an IX83 microscope (Olympus) equipped with an EMCCD camera (Evolve 512, Roper Scientific), a Plan-Apo $100 \times 1.40$ NA oil objective (Olympus) and Metamorph software (Molecular Devices). To change drug concentrations or wash out drugs while performing live-cell imaging, we removed the existing medium in the glass chamber by aspiration and added new medium with or without the respective drug.

\section{Imaging analysis}

For quantification of localization of c-Src wild-type and mutants at focal adhesions (FAs) in XTC cells, we transfected EGFP-N1-C-Src wild-type or mutants along with TagRFP-T-C1-FAK as a marker of FAs, and collected live-cell imaging data of EGFP-fused c-Src wild-type or mutants with TagRFP-T-FAK in the same cells. To detect FA ROls at each frame of data, TagRFP-T-FAK imaging data were analyzed using step-by-step FA detection analysis (Horzum et al., 2014). Images of c-Src-EGFP wild-type and mutants were overlaid onto the detected FA-ROI, and the EGFP intensities in the FA-ROI and in the whole cell area were measured at each frame. To derive the average intensity outside of FAs, total EGFP intensity at FAs was subtracted from total EGFP intensity in the whole cell area, and then divided by the cell area outside of the FAs. To calculate the ratio of c-Src-EGFP intensity at FAs 
to the intensity outside of FAs, after subtraction of background intensities in extracellular regions, the average EGFP intensity at FAs was divided by the average EGFP intensity outside of FAs.

To compare the expression level of c-Src-EGFP, whole-cell EGFP images were acquired before live-cell time-lapse imaging. There was no significant difference between each experiment.

\section{Protein expression and purification}

HEK293F cells were transfected with Flag-6p-C1-6 $\times$ His-Src, Flag-6p-C1-6 $\times$ His-Src -T338I, Flag-6p-C1-c-Src, Flag-6p-C1-c-SrcT338I, Flag-6p-C1-c-Src-K249E-P250E-Y527F, Flag-C1-FAK-411-686 (FAK kinase domain), Flag-C1- $\mathrm{FERM}$ and c-Abl-1-623-EGFP$3 \times$ Flag using polyethyleneimine (PEl). After 2 days, cells were lysed in buffer $\mathrm{A}\left(50 \mathrm{mM}\right.$ Tris- $\mathrm{HCl} \mathrm{pH} 8.0$ at $4^{\circ} \mathrm{C}, 150 \mathrm{mM} \mathrm{NaCl}, 0.2 \mathrm{mM}$ orthovanadate, 1 mM DTT, 1 mM EDTA, 1\% Triton X-100 and protease inhibitor cocktail (nacalai tesque, 03969-34)). After centrifugation, the supernatant was incubated with anti-DYKDDDDK tag antibody beads (Wako, 018-22783) at $4^{\circ} \mathrm{C}$ for 2 hours. Beads were washed four times with buffer $\mathrm{B}\left(20 \mathrm{mM}\right.$ Tris- $\mathrm{HCl} \mathrm{pH} 8.0$ at $4^{\circ} \mathrm{C}, 500 \mathrm{mM} \mathrm{NaCl}, 1 \mathrm{mM}$ EDTA, $1 \mathrm{mM}$ DTT and 1\% Triton X-100). FLAG-6p-6 $\times$ His-Src, FLAG-6p-c-Src, FLAG-6p-c-Src-T338I or FLAG-6p-c-Src- K249E-P250E-Y527F-bound beads were incubated with GST-PreScission Protease (GE Healthcare, 27084301)-bound GST-Accept beads (nacalai tesque, 09277-72) in buffer C (50 mM HEPES-KOH pH 7.4, $50 \mathrm{mM} \mathrm{KCl}, 2 \mathrm{mM} \mathrm{MgCl}_{2}, 0.1 \%$ Tween-20, $0.2 \mathrm{mM}$ orthovanadate and $1 \mathrm{mM} \mathrm{DTT}$ ) at $4^{\circ} \mathrm{C}$ for 18 hours. FLAG- $\Delta$ FERM or c-Abl-1-623-EGFP-3 $\times$ FLAG-bound beads were incubated with purified GST-PTP-1B ${ }^{1-321}$ (see below) in buffer D (20 mM HEPES-NaOH pH7.4, $100 \mathrm{mM} \mathrm{NaCl}, 1 \mathrm{mM} \mathrm{DTT}, 0.1 \%$ Triton X-100 and $5 \mathrm{mM} \mathrm{MnCl}_{2}$ ) for 1 hour at RT. Beads were washed four times with buffer B. To assay autophosphorylation of pY397 in FAK, beads were incubated in buffer E (20 mM HEPES-KOH pH7.4 at RT, $50 \mathrm{mM} \mathrm{KCl}, 1 \mathrm{mM}$ DTT, $0.1 \%$ Triton X-100, $5 \mathrm{mM} \mathrm{MgCl}_{2}, 5 \mathrm{mM}$ ATP (nacalai tesque, 01072-24) and 0.2 mM sodium orthovanadate) for 30 mins at RT. After autophosphorylation, beads were washed four times with buffer B supplemented with 0.2 mM sodium orthovanadate and GST-Accept beads were added to remove GST-PTP-1B ${ }^{1-321}$. FLAG-FAK kinase domain, FLAG- $\triangle$ FERM or C-Abl-1-623EGFP-3 $\times$ FLAG-bound beads were eluted with $1 \mathrm{mg} / \mathrm{ml}$ DYKDDDK peptide (Wako, 044-30951) in buffer F for FLAG-FAK kinase domain (20 mM HEPES-NaOH pH7.4 at RT, $100 \mathrm{mM} \mathrm{NaCl}, 1 \mathrm{mM}$ DTT, 0.1\% Triton X-100, $5 \mathrm{mM} \mathrm{MgCl}$ ( $_{2}$ or buffer C for FLAG- $\Delta$ FERM and c-Abl-1-623-EGFP-3 $\times$ FLAG at $4^{\circ} \mathrm{C}$ for 30 min, respectively. Purified FLAG-FAK kinase domain, FLAG- $\triangle$ FERM and c-Abl-1-623EGFP-3 $\times$ FLAG were dialyzed against buffer $F$ without Triton X-100 and buffer $C$ without Tween-20, respectively.

For bacterial expression of GST, GST-PTP-1B ${ }^{1-321}$, GST-FAK ${ }^{362-411}$, GST-FAK ${ }^{362-411-P 371 A-P 374 A}$ and GST-Abi-1, E. coli BL21(DE3) (NEB, C2527) was used as a host. Induction was carried out for 18 hours with $0.1 \mathrm{mM}$ IPTG at $16^{\circ} \mathrm{C}$. Cells were resuspended in buffer $\mathrm{G}\left(20 \mathrm{mM}\right.$ Tris- $\mathrm{HCl} \mathrm{pH} 8.0$ at $4^{\circ} \mathrm{C}, 150 \mathrm{mM} \mathrm{NaCl}, 1 \mathrm{mM} \mathrm{DTT}, 1 \mathrm{mM}$ EDTA, $0.2 \%$ Triton X-100 and protease inhibitor cocktail) and sonicated. After centrifugation, the supernatant was incubated with GST-Accept beads at $4^{\circ} \mathrm{C}$ for 1 hour. The beads were washed four times with buffer B. GST-PTP-1B ${ }^{1-321}$ was eluted with buffer $\mathrm{H}\left(100 \mathrm{mM} \mathrm{Tris}-\mathrm{HCl} \mathrm{pH} 8.0\right.$ at $4^{\circ} \mathrm{C}, 150 \mathrm{mM} \mathrm{NaCl}$, $1 \mathrm{mM}$ DTT, $0.1 \%$ Triton $\mathrm{X}-100$ and $20 \mathrm{mM}$ glutathione) for $30 \mathrm{~min}$ at $4{ }^{\circ} \mathrm{C}$, and dialyzed against dialysis buffer $\mathrm{D}$ without Triton $\mathrm{X}-100$. GST, GST-FAK ${ }^{362-411}$ and GST-Abi-1 were eluted with buffer I (100 mM Tris-HCl pH 8.0, $50 \mathrm{mM} \mathrm{KCl,} 1 \mathrm{mM}$ DTT, 0.1\% Tween-20, $0.2 \mathrm{mM}$ sodium orthovanadate and $20 \mathrm{mM}$ glutathione) for $30 \mathrm{~min}$ at $4{ }^{\circ} \mathrm{C}$ and dialyzed against buffer $\mathrm{C}$ without Tween-20. For phosphorylation of Y397 in GST-FAK ${ }^{362-411}$ or Y397 in GST-FAK ${ }^{362-411-P 371 A-P 374 A, ~ p r o t e i n-b o u n d ~ b e a d s ~ w e r e ~ i n c u b a t e d ~ w i t h ~ p u-~}$ rified FLAG-FAK kinase domain in buffer F with $10 \mathrm{mM}$ ATP at RT for 1 hour. Phosphorylated GST-FAK ${ }^{362-411}$ or phosphorylated GST-FAK ${ }^{362-411-P 371 A-P 374 A}$-bound beads were washed four times with buffer B and eluted with buffer I (100 mM Tris-HCl pH 8.0 , $50 \mathrm{mM} \mathrm{KCl}, 1 \mathrm{mM}$ DTT, $0.1 \%$ Tween-20, $0.2 \mathrm{mM}$ sodium orthovanadate and $20 \mathrm{mM}$ glutathione) for 30 min at $4{ }^{\circ} \mathrm{C}$. Purified phosphorylated GST-FAK ${ }^{362-411}$ was dialyzed against buffer $\mathrm{C}$ without Tween-20.

\section{AlphaScreen in vitro binding assay}

$6 \times$ His-c-Src or $6 \times$ His-c-Src-T338I recombinant protein $(50 \mathrm{nM})$ was mixed with $0.5 \mu \mathrm{g}$ of Protein A acceptor beads (PerkinElmer, 6760137) and 1:500 diluted anti-His antibodies (MBL, PM032) in $10 \mu \mathrm{L}$ binding buffer A (50 mM HEPES-KOH pH 7.4, $50 \mathrm{mM} \mathrm{KCl,} 2 \mathrm{mM}$ $\mathrm{MgCl}_{2}, 0.1 \%$ Tween-20, 0.05\% ovalbumin (Sigma Aldrich, A5503), $1 \mathrm{mM} \mathrm{DTT}$ and $0.2 \mathrm{mM}$ sodium orthovanadate). Separately, purified GST-phosphorylated Y397-FAK ${ }^{362-411}$, GST-FAK ${ }^{362-411}$, GST-phosphorylated Y397-FAK ${ }^{362-411-P 371 A-P 374 A}$ or GST (50 nM) proteins were mixed with $0.5 \mu \mathrm{g}$ glutathione-donor beads (PerkinElmer, 6765301) in $10 \mu$ l. The mixtures were incubated for 30 min at RT. Kinase inhibitors in $5 \mu \mathrm{L}$ of binding buffer $\mathrm{A}$ and the aforementioned $10 \mu \mathrm{L}$ mixtures of Acceptor beads were combined in wells of a $384-$ well Optiplate (PerkinElmer, 6007290) and incubated for $15 \mathrm{~min}$ at RT. The mixtures of Donor beads were then added to the kinase inhibitors and the Protein A acceptor mixtures in the 384-well Optiplate and further incubated for 1 hour at RT. The final concentrations of inhibitors in each well were those described in the figures. The emission signal was monitored using EnVision (PerkinElmer). For in vitro binding of c-Abl to Abi-1, c-Abl-1-623-EGFP-3 x FLAG, purified recombinant protein (50 nM) was mixed with $0.5 \mu \mathrm{g}$ of Protein A acceptor beads and 1:1000 diluted anti-GFP antibodies (Abcam, ab290) in $10 \mu \mathrm{L}$ binding buffer B (50 mM HEPES-KOH pH 7.4, $50 \mathrm{mM} \mathrm{KCl}, 4 \mathrm{mM} \mathrm{MgCl}$, $0.1 \%$ Tween-20, $0.05 \%$ BSA (Sigma Aldrich, A7030) and $0.2 \mathrm{mM}$ sodium orthovanadate). Separately, purified GST-Abi-1 or GST $(50 \mathrm{nM})$ proteins were mixed with $0.5 \mu \mathrm{g}$ GST-donor beads in $10 \mu \mathrm{L}$ buffer B. Detailed methods were the same as above, but using buffer B. The $\mathrm{EC}_{50}$ values of inhibitors for $\mathrm{c}-\mathrm{Src}$ and c-Abl were calculated using GraphPad Prism 6.

\section{In vitro kinase assay}

For the inhibitor-washout c-Src activation assay, purified FLAG- $\Delta$ FERM was incubated with anti-DYKDDDDK tag antibody beads in binding buffer (20 mM HEPES-KOH pH 7.4, $50 \mathrm{mM} \mathrm{KCl,} 2 \mathrm{mM} \mathrm{MgCl}, 0.1 \%$ Tween-20, $0.2 \mathrm{mM}$ sodium orthovanadate and $1 \mathrm{mM}$ 
DTT) for $1 \mathrm{hr}$ at RT. FLAG- $\Delta$ FERM-bound beads were washed four times with binding buffer and washed two times with reaction buffer (20 mM HEPES-KOH pH 7.4, $50 \mathrm{mM} \mathrm{KCl,} 2 \mathrm{mM} \mathrm{MgCl}_{2}, 0.1 \%$ Tween-20, $0.5 \%$ ovalbumin, 0.2 mM sodium orthovanadate and $1 \mathrm{mM} \mathrm{DTT)}$. FLAG- $\triangle$ FERM-bound beads were incubated with c-Src wild-type recombinant protein, inhibitors and 2 mM ATP in spin columns (Thermofisher, 26147) for 2 or $7 \mathrm{~min}$ at RT. For inhibitor-washout, after 2 min of the above reaction with ATP, samples were centrifuged at $1,000 \mathrm{~g}$ for $30 \mathrm{~s}$ at RT and the beads were incubated with $2 \mathrm{mM}$ ATP in reaction buffer. Reactions were stopped by adding SDS sample buffer and incubated for $10 \mathrm{~min}$ at RT. samples were centrifuged at 1,000 $\mathrm{g}$ for $30 \mathrm{~s}$ at RT and boiled. Two hundred ng of FLAG- $\triangle$ FERM recombinant proteins and $30 \mathrm{ng}$ of Src recombinant proteins were used in each reaction.

For drug-resistant c-Src activation assays, purified FLAG- $\Delta$ FERM was incubated with anti-DYKDDDDK tag antibody beads in binding buffer for $1 \mathrm{hr}$ at RT. FLAG- $\triangle$ FERM-bound beads were washed four times with binding buffer and washed two times with reaction buffer. FLAG- $\Delta$ FERM-bound beads were incubated with c-Src wild-type or T338I mutant recombinant proteins, inhibitors and $2 \mathrm{mM}$ ATP for $10 \mathrm{~min}$ at RT (200 ng FLAG- $\Delta$ FERM recombinant protein and $30 \mathrm{ng}$ Src recombinant protein were used for each reaction). After the reaction, SDS-sample buffer was added to the samples, and the samples were boiled.

\section{Western blotting}

For western blot analysis of the washout of inhibitors, MCF-7 cells were starved in DMEM for 24 hours. After 10 min of treatment with DMSO or inhibitors, the medium was removed, and cells were washed twice with DMEM. After washing, cells were incubated with DMEM for 15 mins. After stimulation, the medium was removed, and cells were washed with cold PBS. Cells were lysed in lysis buffer (50 mM Tris- $\mathrm{HCl} \mathrm{pH} 7.5$ at $4^{\circ} \mathrm{C}, 150 \mathrm{mM} \mathrm{NaCl}, 1 \%$ Triton $\mathrm{X}-100,0.1 \%$ SDS, $0.5 \%$ sodium deoxycholate, $1 \mathrm{mM}$ DTT, $1 \mathrm{mM}$ sodium orthovanadate, $5 \mathrm{mM} \mathrm{NaF}$ (Wako, 192-01972) and protease inhibitor cocktail) and sonicated on ice. After centrifugation at 20,000 $\mathrm{g}$ for $15 \mathrm{~min}$ at $4^{\circ} \mathrm{C}$, the supernatant was collected and subjected to SDS-PAGE.

For western blot analysis of MCF-7-C-Src-T341I and MCF-7 cells, after treatment with inhibitors, the medium was removed, and cells were washed with cold PBS. Cells were lysed in lysis buffer and sonicated on ice. After centrifugation at 20,000 $\mathrm{g}$ for $15 \mathrm{~min}$ at $4{ }^{\circ} \mathrm{C}$, the supernatant was collected and subjected to SDS-PAGE. The following antibodies were used for western blotting: antipY416-Src (1:1,000, Cell Signaling Technology, 2101), anti-non-phospho-Y527-Src (unphosphorylated Y527-Src) (1:1,000, Cell Signaling Technology, 2107), anti-Src (1:1,000, Cell Signaling Technology, 2110) for Figures 4D, 5E, S4E, and S5B, anti-Src (1:1,000, Cell Signaling Technology, 2108) for Figures 4A, 5A, and S4A, anti-pY925-FAK (1:1,000, abcam, ab38512), anti-FAK (1:1,000, Cell Signaling Technology, 3285), anti-Erk (1:2,000, Sigma-Aldrich, M7927) and anti-pErk (1:2,000, Cell Signaling Technology, 9106).

\section{Proliferation assay}

MCF-7 and MCF-7-C-Src-T341I cells were seeded at a density of $1 \times 10^{4}$ cells per well in 24-well plates and grown for 24 hours at $37^{\circ} \mathrm{C}$. Cells were then treated with kinase inhibitors or DMSO for 4 days. After removal of the medium, cells were fixed with $4 \%$ PFA in PBS for $15 \mathrm{~min}$ at RT. Cells were stained with $0.1 \%$ Crystal Violet in $20 \%$ methanol for 10 min at RT. Crystal Violet was extracted with $1 \%$ SDS in distilled water overnight, and the absorbance was measured at $595 \mathrm{~nm}$ using EnVision.

\section{QUANTIFICATION AND STATISTICAL ANALYSIS}

Data and statistical analyses were performed using GraphPad Prism 6, ImageJ and Excel. All numeric data are shown as mean \pm SEM and numbers per group are represented in legends of each panel. 
Cell Reports, Volume 34

Supplemental information

Paradoxical activation of c-Src

as a drug-resistant mechanism

Makio Higuchi, Kenichi Ishiyama, Masahiro Maruoka, Ryosuke Kanamori, Akifumi Takaori-Kondo, and Naoki Watanabe 


\section{Supplemental Information}


A
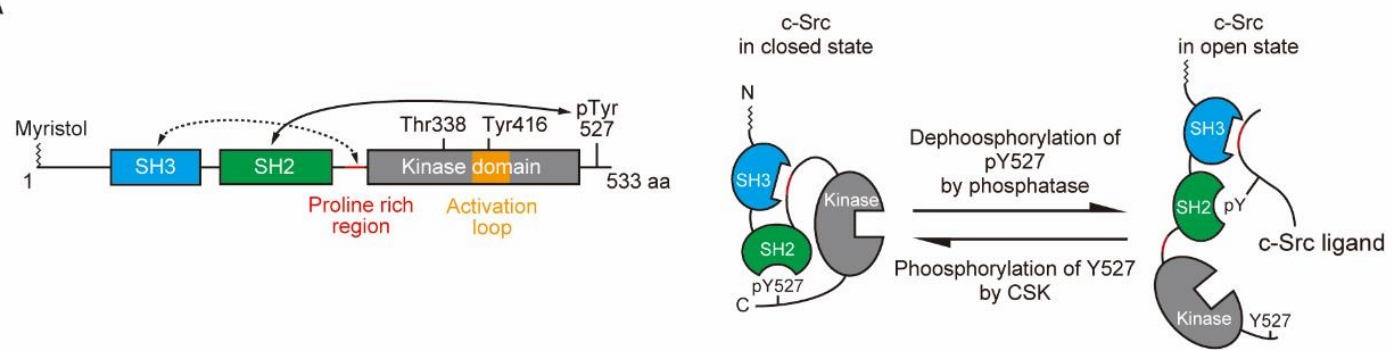

B
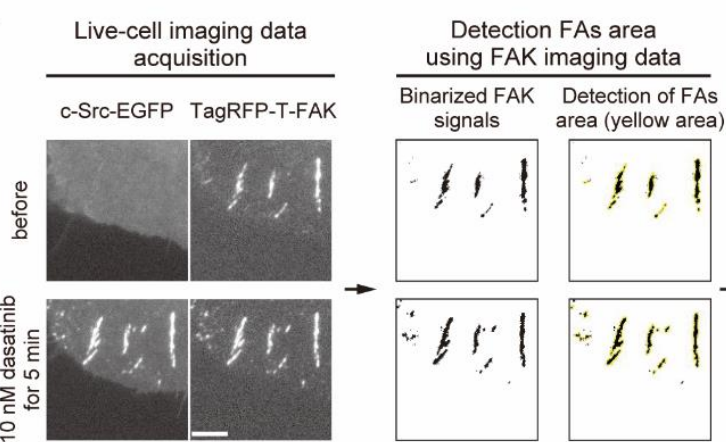

Measurement of intensity and area of c-Src-EGFP
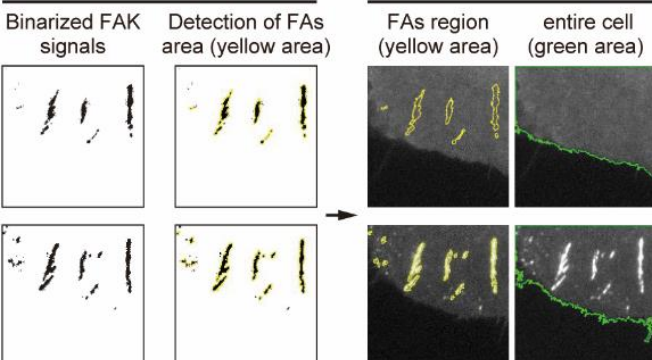
(yellow area) (green area)
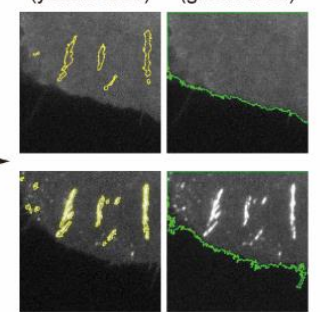

C
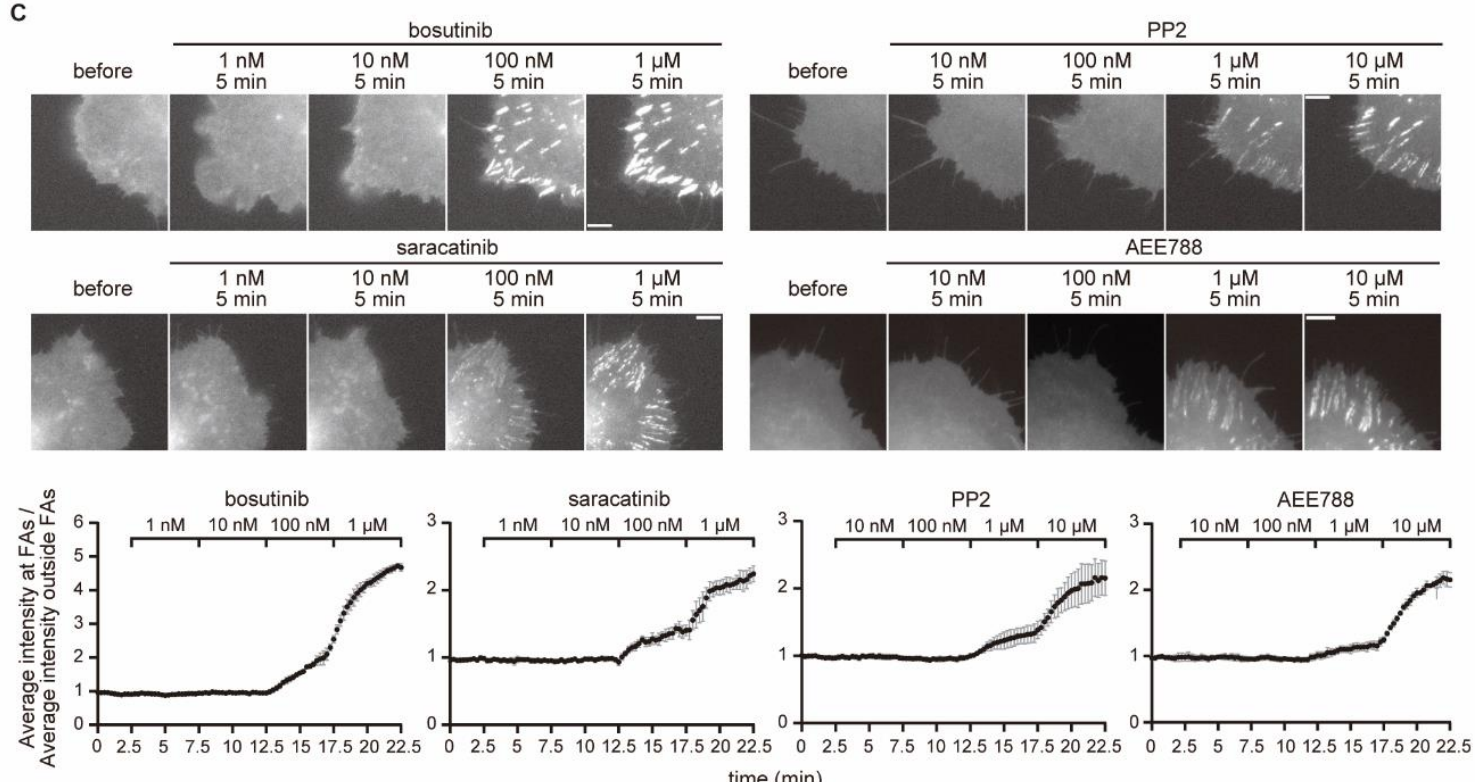
time ( $\mathrm{min})$

Figure S1. Src Inhibitors and RTK Inhibitors Induce Translocation of c-Src to Focal Adhesions.

Related to Figure 1 and Figure 2. (A) The structure of c-Src and a schematic of auto-inhibitory regulation.

c-Src consists SH3, SH2 and tyrosine kinase domains. Myristoylation of glycine at the N-terminus anchors c-Src on the plasma membrane. SH3 and SH2 domains bind the proline-rich motif and phosphotyrosine, respectively. These two regions are crucial for autoinhibition and ligand binding of c-Src. Tyrosine 416 in the activation loop of kinase domain is auto-phosphorylated, which enhances the kinase activity. Several mutations of threonine 338 (gatekeeper mutations) in kinase domain confer to inhibitor-resistance of c-Src. Tyrosine 527 in C-terminal is phosphorylated by C-terminal Src kinase (CSK), which contributes to autoinhibition (left). The SH3 domain binds the linker between SH2 and Kinase domains which contains a 
proline-rich motif. The SH2 domain binds phosphorylated tyrosine at 527 in the C-terminus. These two mechanisms keep c-Src in a closed, autoinhibited state. This autoinhibition is relieved by dephosphorylation of phosphotyrosine at 527, which allows c-Src to bind partner molecules (right). T338, Y416 and Y527 in chicken c-Src correspond to T341, Y419 and Y530 in human c-Src. (B) The schematic of quantification of localization of c-Src at focal adhesions (FAs). To detect FA regions, c-Src-EGFP wild-type or mutants were coexpressed with TagRFP-T-FAK. Images of FAK were binarized and FA regions were defined (see methods for detail). Fluorescence intensity of c-Src-EGFP wild-type or mutants in the detected FA regions (yellow) and the intensity in the entire cell area (outlined by green lines) at each frame were measured after subtraction of background values outside of the cell image. The ratio was calculated by dividing the average fluorescence intensity in FA regions by the average intensity in the cell area outside of FA regions. (C) Src inhibitors, bosutinib, saracatinib, PP2 and RTK inhibitor NVP-AEE788 (AEE788) induce translocation of c-Src-EGFP to FAs. The drug concentration was increased every $5 \mathrm{~min}$. The graphs show that the ratio of the fluorescence intensity of c-Src-EGFP in FA regions and in the cell area outside of FAs. Data are presented as the mean $\pm \operatorname{SEM}(n=3)$. Scale bars, $5 \mu \mathrm{m}$. 
A

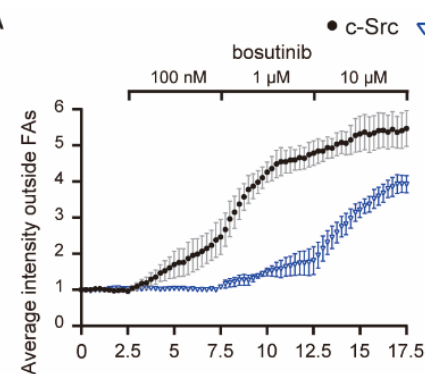

c-Src $\nabla$ C-Src-T338I
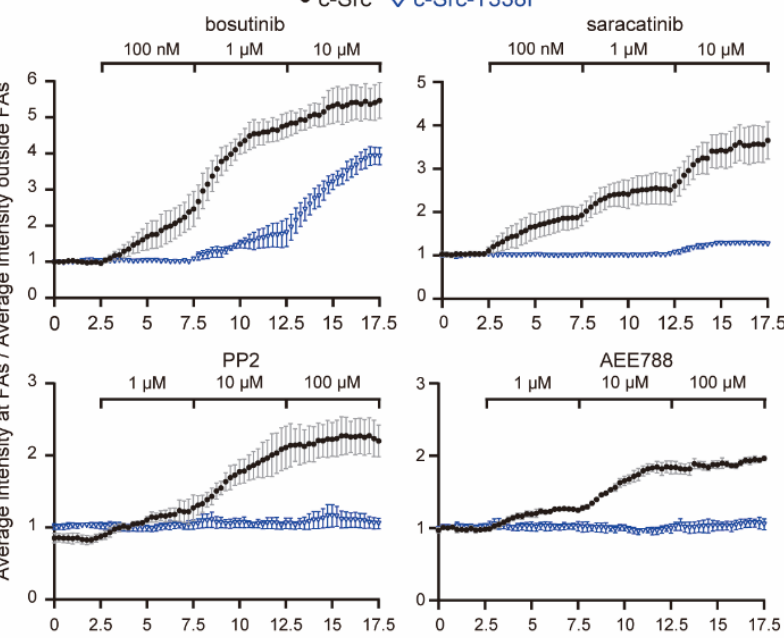

time (min)

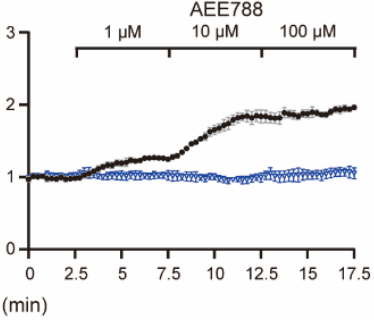

B

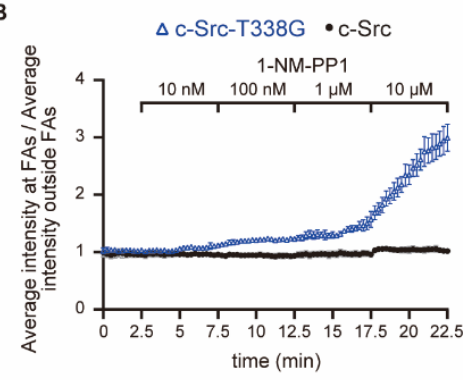

time (min)

C

OG2A $\square$ W118A $\triangle$ R175A $\nabla$ K295M $\diamond K 249 E-P 250 E-Y 527 F \bullet W 118 A-R 175 A$
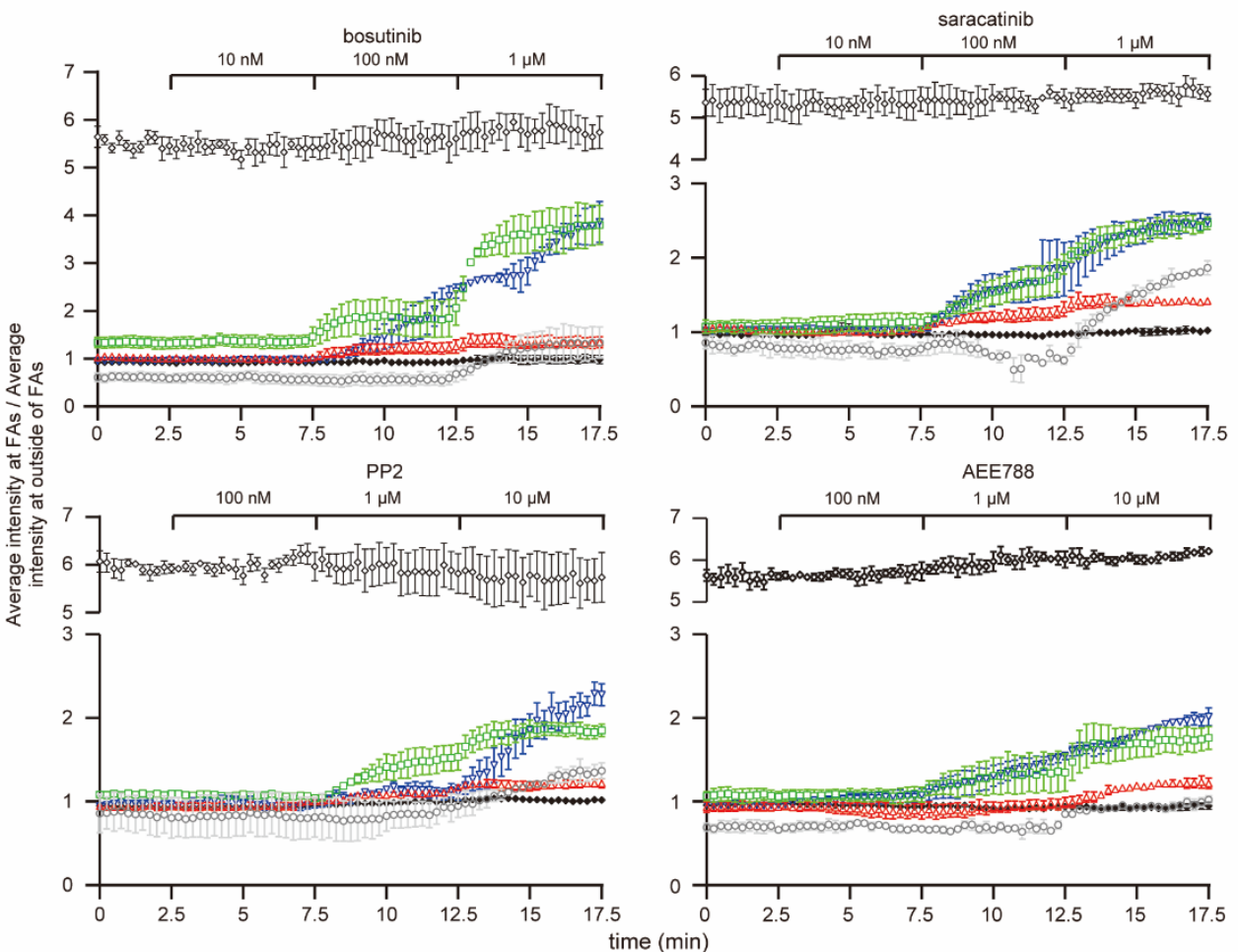

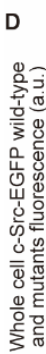

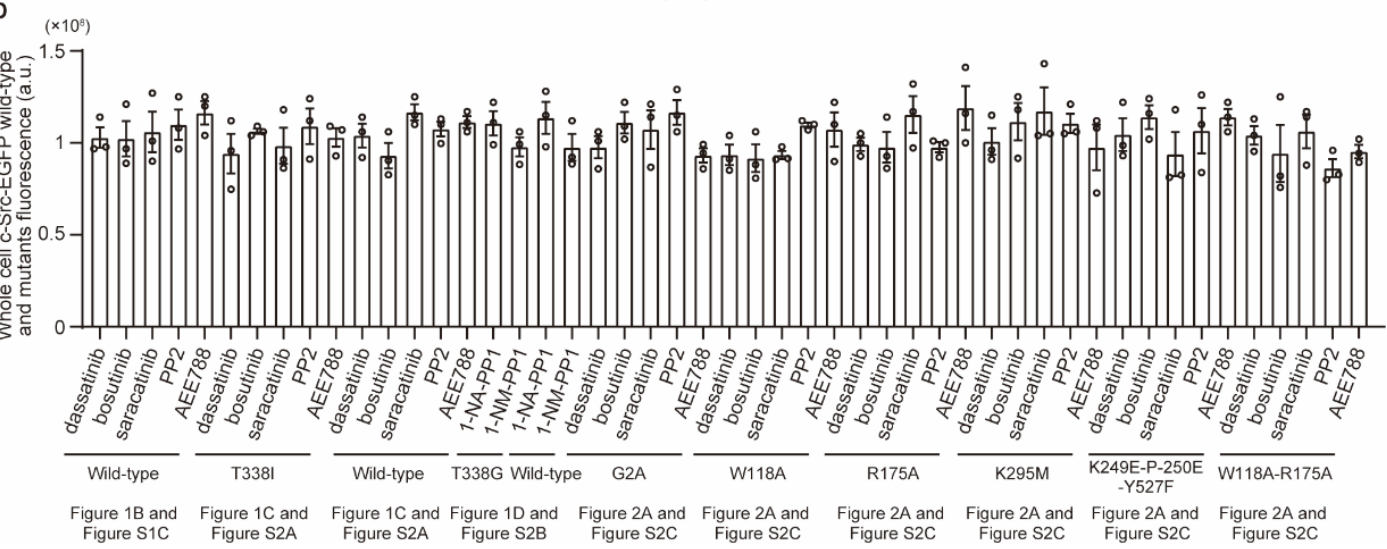


Figure S2. Inhibitor-induced Localization of c-Src-EGFP Function-deficient Mutants. Related to Figure 1 and Figure 2. (A) Quantitative analysis of localization of drug-resistant c-Src-T338I-EGFP or wild-type c-Src-EGFP at FAs during treatment with bosutinib, saracatinib, PP2 and NVP-AEE788 (AEE788). The graphs show that the ratio of EGFP fluorescence intensity in FA regions and in the cell area outside of FAs. Data are presented as the mean \pm SEM $(n=3)$. (B) Quantitative analysis of localization of analog-sensitive c-Src-T338G-EGFP or wild-type c-Src-EGFP at FAs during treatment with 1-NM-PP1. Data are presented as the mean $\pm \operatorname{SEM}(n=3)$. (C) Quantitative analysis of localization of function-deficient c-Src-EGFP mutants, G2A, W118A, R175A, K295M, K249E-P250E-Y527E and W118A-R175A at FAs during treatment with bosutinib, saracatinib, PP2 and NVP-AEE788 (AEE788). Data are presented as the mean \pm SEM ( $n=3)$. (D) Comparison of the expression level of c-Src-EGFP and its mutants in the cells observed in this study. The total intensity of EGFP fluorescence in the whole cell areas are shown for each cell. Data are presented as the mean \pm SEM $(n=3)$. There was no significant difference between each experiment. 
A<smiles>Cc1ccc(NC(=O)c2ccc(CN3CCN(C)CC3)cc2)cc1Nc1nccc(-c2cccnc2)n1</smiles>
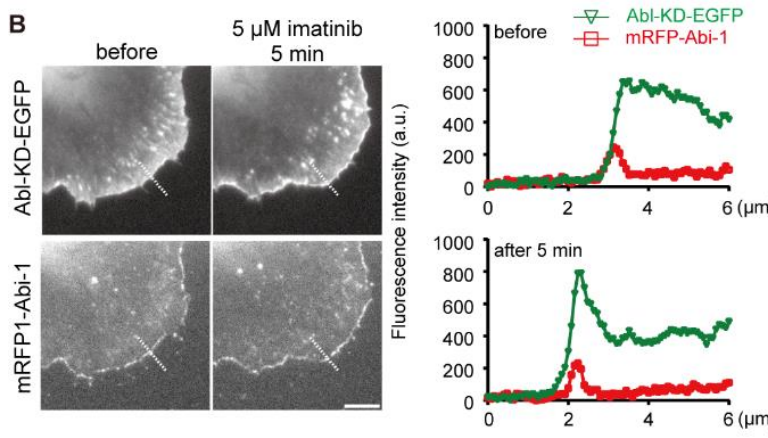

D
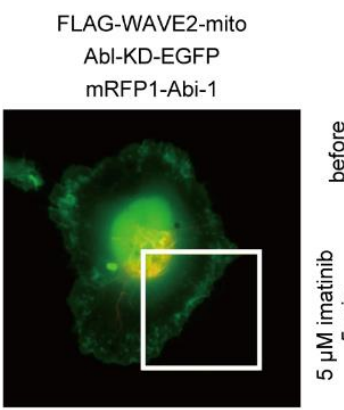

C

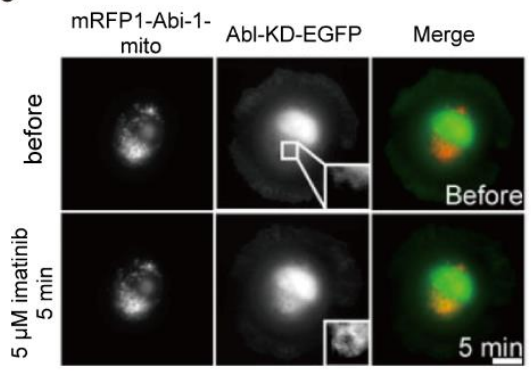

E

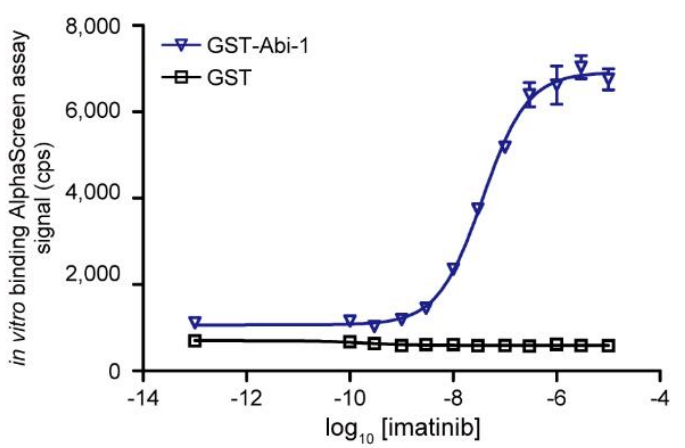

Figure S3. ATP-competitive inhibitors relieve auto-inhibition of target kinase and lead to binding of the kinase to partner molecule. Related to Figure 3. (A) A chemical structure of imatinib. (B) Imatinibinduced colocalization of kinase deficient point mutant Abl-K290M-EGFP (Abl-KD-EGFP) and mRFP1Abi-1 at the lamellipodium tip of XTC cells. Images before and $5 \mathrm{~min}$ after $5 \mu \mathrm{M}$ imatinib treatment are shown. After imatinib treatment, Abl-KD showed overlapping distribution with Abi-1 at the cell edge and dot-like puncta. The graphs show fluorescence intensity of Abl-KD (green) and Abi-1 (red) along the dotted line. Scale bar, $5 \mu \mathrm{m}$. (C) Imatinib at $5 \mu \mathrm{M}$ induced accumulation of Abl-KD-EGFP (green) on mitochondria of cells coexpressing mRFP1-Abi-1-mito (red). Images before and 5 min after $5 \mu \mathrm{M}$ imatinib treatment are shown. Magnified views of the box region are shown in the inset. Scale bar, $5 \mu \mathrm{m}$. (D) FLAGWAVE2-mito, Abl-KD-EGFP (green) and mRFP1-Abi-1 (red) were coexpressed in XTC cells. Depletion of Abi-1 from the cell periphery was monitored by the localization of mRFP1-Abi-1. Images before and 5 min after $5 \mu \mathrm{M}$ imatinib treatment are shown. Images of Abi-1 and Abl-KD at the cell periphery (square) before and after imatinib treatment are shown on the right. Scale bar, $5 \mu \mathrm{m}$. (E) In vitro AlphaScreen binding assay of purified recombinant c-Abl-EGFP-3×FLAG with GST-tagged Abi-1 or GST. Imatinib enhanced the binding of c-Abl and Abi-1 in a dose-dependent manner. The binding assay was monitored by the AlphaScreen assay. Data are presented as the mean $\pm \operatorname{SEM}(n=3) . \mathrm{EC}_{50}$ value for imatinib was $37 \mathrm{nM}$. 
A

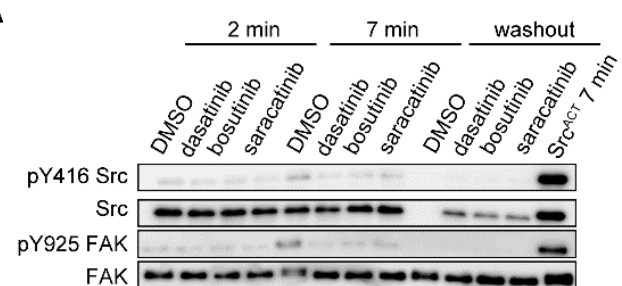

C
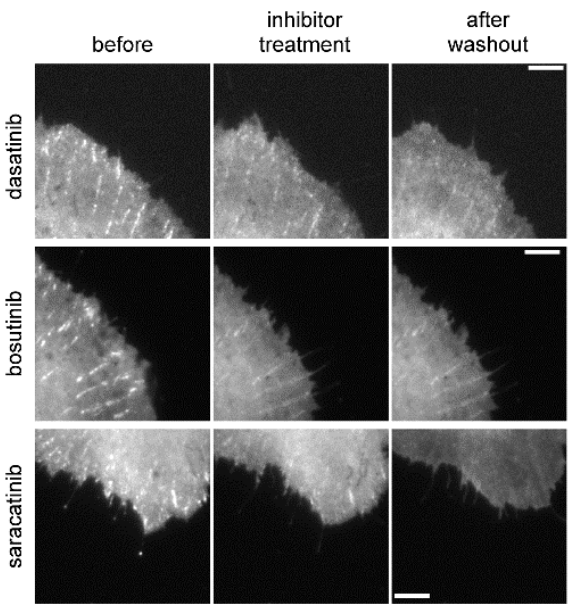

B

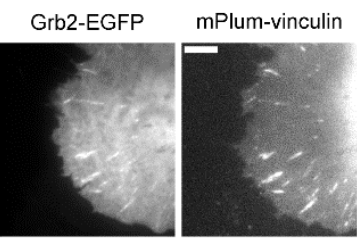

D
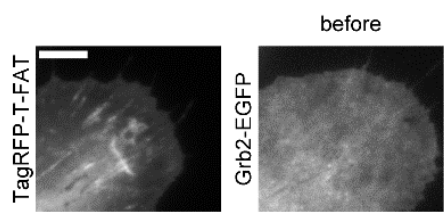

$10 \mu \mathrm{M}$ PP2

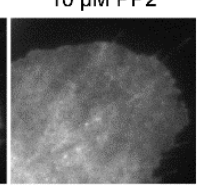

$10 \mathrm{~min}$

$30 \mathrm{~min}$

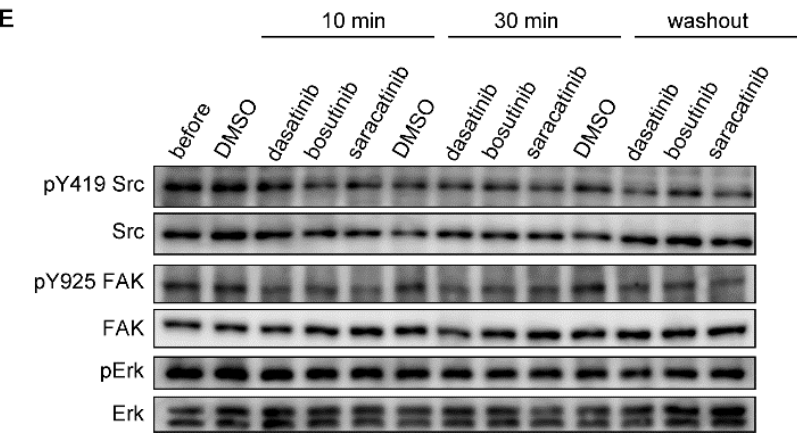

Figure S4. Treatment and subsequent washout of high affinity Src inhibitors do not activate c-Src and FAK-Grb2-Erk signaling. Related to Figure 4. (A) Western blot for phospho-Y416 Src, rabbit polyclonal Src, pY925 FAK and FAK of in vitro kinase assay of c-Src and FAK with treatment and washout of high affinity Src inhibitors. In lanes indicated by "washout", after stimulation of $0.1 \%$ DMSO, $100 \mathrm{nM}$ dasatinib, $1 \mu \mathrm{M}$ bosutinib or $1 \mu \mathrm{M}$ saracatinib with $2 \mathrm{mM}$ ATP for $2 \mathrm{~min}$, washout was carried out and samples were incubated for $5 \mathrm{~min}$ after the addition of $2 \mathrm{mM}$ ATP. Src ${ }^{\mathrm{ACT}}$ means c-Src-K249E-P250EY527F. (B) Grb2-EGFP associates with FAs. Images show the localization of Grb2-EGFP and mPlumvinculin in XTC cells. mPlum-vinculin was used as a marker for FAs. Scale bar, $5 \mu \mathrm{m}$. (C) Live-cell imaging of Grb2-EGFP during treatment and subsequent washout of high affinity Src inhibitors. $100 \mathrm{nM}$ dasatinib, $1 \mu \mathrm{M}$ bosutinib and $1 \mu \mathrm{M}$ saracatiinib were used. Images before, 5 min after drug treatment and $10 \mathrm{~min}$ after washout are shown. The treatment was performed for $5 \mathrm{~min}$. Scale bars, $5 \mu \mathrm{m}$. (D) The localization and translocation of Grb2 to FAs are dependent on the localization of FAK at FAs. Overexpression of the FAT domain of FAK, which depletes FAK from FAs (Figure 3B), abrogated the association of Grb2-EGFP with FAs before drug treatment. Treatment and washout of PP2 did not induce an increase in Grb2-EGFP signals at FAs. Images before, 5 min after PP2 treatment and 10 min after washout are shown. Scale bar, $5 \mu \mathrm{m}$. (E) Western blot for phospho-Y419 Src, mouse monoclonal Src, phospho-Erk (pErk) and Erk1\&2 (Erk) in MCF-7 cells with treatment and washout of inhibitors. After 
stimulation with $0.1 \%$ DMSO, $100 \mathrm{nM}$ dasatinib, $1 \mu \mathrm{M}$ bosutinib and $1 \mu \mathrm{M}$ saracatiinib for 10 min, washout was carried out and incubated for $20 \mathrm{~min}$. 
A

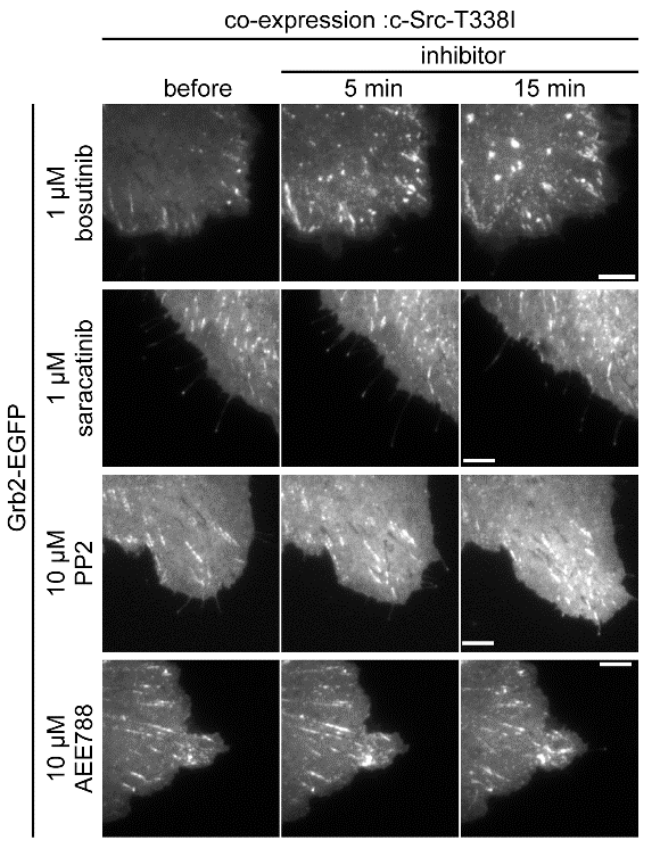

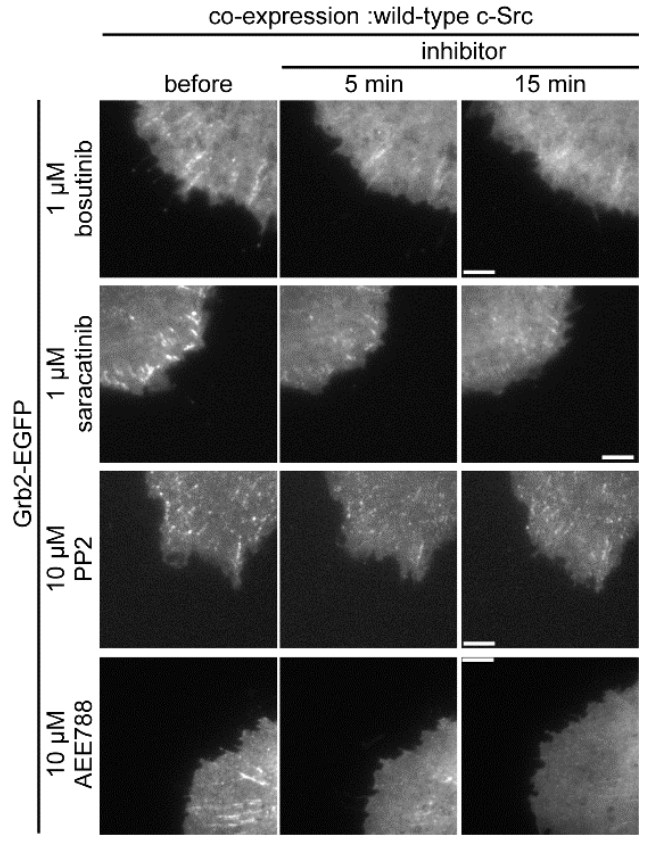

B

MCF-7-T341।
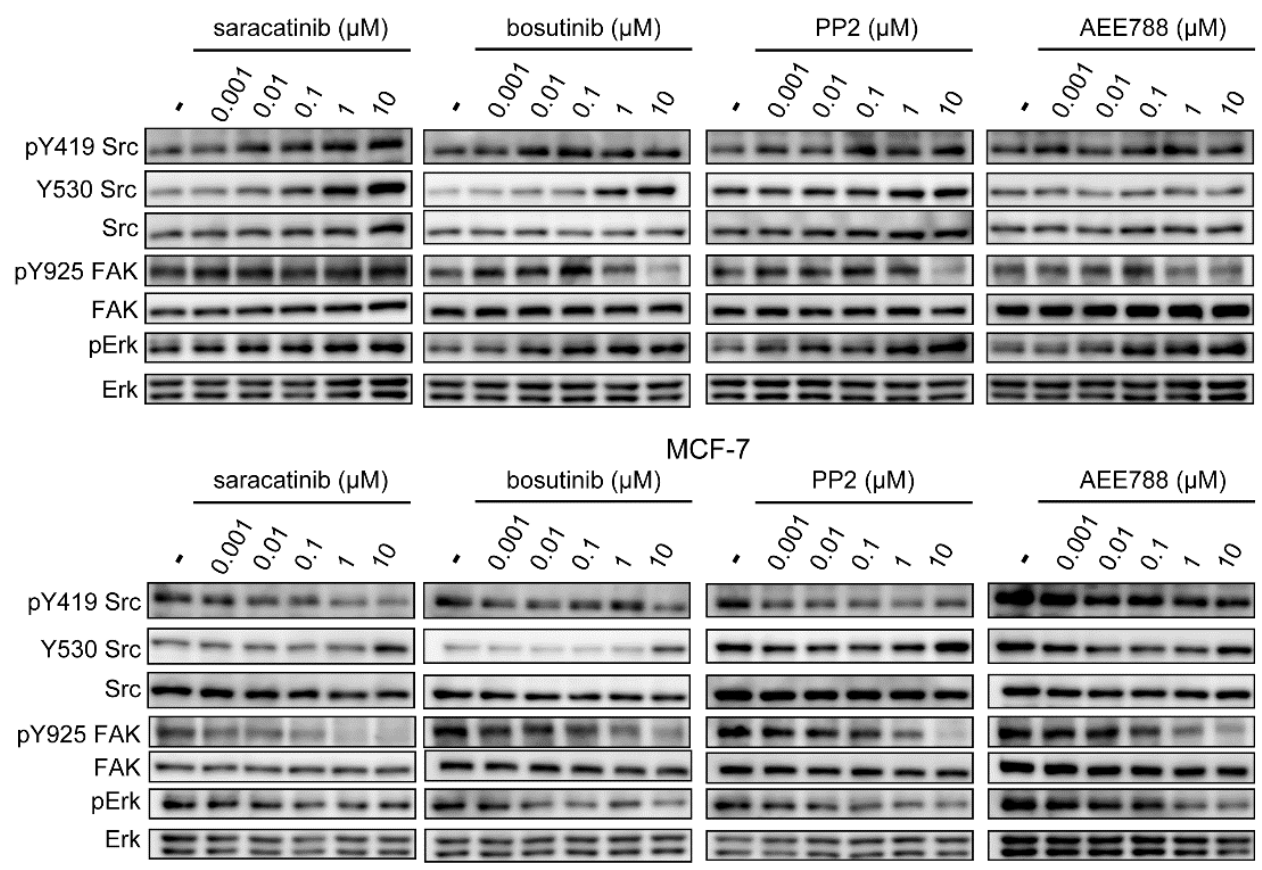

Figure S5. Src Kinase Inhibitors Activate c-Src-FAK-Grb2-Erk Signaling in the presence of drug resistant c-Src mutant. Related to Figure 5. (A) Bosutinib, saracatinib, PP2 and NVP-AEE788 (AEE788) activate c-Src-T338I in cells. Treatment of bosutinib, saracatinib, PP2 and AEE788 induced translocation of Grb2-EGFP to FAs in XTC cells expressing c-Src-T338I-TagRFP-T, whereas the treatment decreased the localization of Grb2-EGFP in XTC cells expressing wild-type c-Src-TagRFP-T. Images before, 5 min and 15 min after drug treatment are shown. Scale bars, $5 \mu \mathrm{m}$. (B) Western blot for phospho-Y419 Src, 
unphosphorylated Y530 Src, mouse monoclonal Src, pY925-FAK, FAK, phospho-Erk (pErk) and Erk1\&2 (Erk) in MCF-7-c-Src-T341I and MCF-7 cell lines treated with bosutinib, saracatinib, PP2, NVP-AEE788 (AEE788) or $0.1 \%$ DMSO (indicated by “-”) for $30 \mathrm{~min}$. 


\begin{tabular}{|c|c|c|c|c|c|c|c|}
\hline Kinase & Dasatinib & Bosutinib & Saracatinib & PP2 & AEE788 & 1-NA-PP1 & 1-NM-PP1 \\
\hline c-Src & $0.21,0.8^{*}$ & $1,1.2^{*}$ & $2.7^{*}$ & $36^{*}$ & $61^{*}$ & $900^{*}$ & $1200^{*}$ \\
\hline c-Src-T338I & $3,000^{*}$ & 225 & & & & & \\
\hline c-Src-T338G & & & & & $2 *$ & $1.5^{*}$ & $2^{*}$ \\
\hline FAK & 120 & 3.4 & & & $6^{*}$ & & \\
\hline Her2 & 1400 & 0.59 & & & $77^{*}$ & & \\
\hline VEGFR2 & 2,900 & 4.2 & & & & & \\
\hline B-Raf & 3,500 & 3 & & & & & \\
\hline C-Raf & 570 & $>10,000$ & & & & & \\
\hline Mek1 & 1,000 & 19 & & & & & \\
\hline Mek2 & 1,400 & 9.9 & & & & & \\
\hline Erk1 & $>10,000$ & $>10,000$ & & & & & \\
\hline Erk2 & $>10,000$ & $>10,000$ & & & & & \\
\hline
\end{tabular}

Table S1. Reported $K_{\mathrm{d}}$ and $\mathrm{IC}_{50}$ of kinase inhibitors determined in vitro. Related to Figure 1, 3, 4, 5, S1 and S2.

Reported $K_{\mathrm{d}}$ of dasatinib (Karaman et al., 2008), bosutinib (Karaman et al., 2008; Levinson and Boxer, 2014) and reported $\mathrm{IC}_{50}$ of dasatinib (Apsel et al., 2008; O'Hare et al., 2005), bosutinib (Boschelli et al., 2001), saracatinib (Green et al., 2009), PP2 (Bain et al., 2007), AEE788 (Traxler et al., 2004), 1-NA-PP1 (Zhang et al., 2013) and 1-NM-PP1 (Zhang et al., 2013) for c-Src, FAK and Erk signaling-related molecules in vitro. Asterisks indicate $\mathrm{IC}_{50}$ of inhibitors and others indicate $K_{\mathrm{d}}$ of inhibitors. 